\title{
Site chronology and a review of radiocarbon dates from Fiji
}

\author{
Geoffrey Clark \\ Archaeology and Natural History, The Australian National University \\ Atholl Anderson \\ Archaeology and Natural History, The Australian National University
}

\section{Introduction}

The earliest radiocarbon dates from the Central Pacific were obtained by Edward W. Gifford, on charcoal recovered from excavations at Vunda and Navatu on Viti Levu (Gifford 1951a, b), and the results were later used to outline the first culture sequence proposed for Fiji, by Roger Green (1963). Subsequent investigations by Frost (1970, 1979) and Best (1984) substantially increased the number of ${ }^{14} \mathrm{C}$ results from the archipelago, and allowed a wider range of cultural attributes, such as settlement location, interaction pattern, subsistence economy and stone-tool types, to be age-correlated.

This chapter is divided into two sections, with the first section reporting the radiocarbon and thermoluminescence dates obtained by the Early Prehistory of Fiji project (the EPF) between 1996 and 2000. There were 68 radiocarbon determinations from 13 prehistoric sites. Most dated deposits were from coastal or near-coast locations on Viti Levu $(n=9)$, two sites were from Beqa Island, and two excavations were on Mago Island in the Lau Group. Six sites contained ceramics of Lapita style (some mixed with pottery of post-Lapita age), two sites had deposits of mid-sequence antiquity, and five sites had pottery and other items common in late-prehistoric sites dating to the last millennium. Results were used to construct a chronology for each site by assessing the reliability of each determination. In several deposits, the age of the oldest cultural remains was not able to be determined with radiocarbon because of extensive reworking of Lapita remains with more recent cultural materials. 
There are now more than 300 radiocarbon determinations from prehistoric sites in Fiji, and the second section of this chapter presents age results identified in a review of the archaeological literature. In listing the ${ }^{14} \mathrm{C}$ results from Fiji our purpose is to provide a comprehensive overview of archaeological determinations, and to illustrate the geographic locations and points in the Fiji culture sequence that have been the focus of chronometric research. Recent investigations with a significant dating component have greatly altered the temporal dimensions of key sections of the Fijian sequence. These include, in addition to the EPF, the work of Nunn, Kumar and colleagues at Lapita sites (2004a, 2004b), the analysis of mid-sequence ceramic change at the Sigatoka Sand Dunes (Burley 2005), and the study of fortifications (Field 2004, 2005). Our review concludes with a discussion of these parts of the sequence in light of new chronological data.

\section{Site chronology}

Radiocarbon determinations were obtained on samples from two north-coast Viti Levu sites (Natunuku, Navatu 17A), seven southwest Viti Levu sites (Karobo, Malaqereqere, Tuvu, Volivoli II, Volivoli III, Qaranioso I and Qaranioso II), Beqa Island and Ugaga Island (Kulu and Ugaga), and two sites on Mago Island in the Lau Group (Votua and Sovanibeka). There were 68 determinations, two AMS and the rest radiocarbon, and nine samples were 'modern' (Table 17). Two thermoluminescence determinations were obtained on pottery excavated from the Navatu 17A site (Table 18). At the Sigatoka Sand Dunes, an intact cultural deposit was not located, and optically stimulated luminescence (OSL) age results were obtained on the dune sediments (see Anderson et al. 2006). New radiocarbon dates from the Yanuca Lapita site excavated in the 1960s by Lawrence and Helen Birks have been reported previously (Clark and Anderson 2001b), and are listed in the review of archaeological dates (see below).

Radiocarbon samples of marine shell, terrestrial shell, bone, palm wood and charcoal were analysed at the Quaternary Dating Research Centre (QDRC) at the Australian National University ( $\mathrm{n}=45$ ), the Waikato Radiocarbon Dating Laboratory at the University of Waikato $(\mathrm{n}=15)$, the Beta Analytic Inc. Radiocarbon Dating Laboratory (BETA) in Florida ( $\mathrm{n}=7)$, and the Australian Nuclear Sciences and Technology Organisation (ANSTO) at Lucas Heights $(\mathrm{n}=1)$. Two thermoluminescence dates were determined at the Department of Physics at the University of Adelaide. Sample pretreatment and calibration are outlined below. Radiocarbon results from the EPF are given in Table 17 and Figure 83, with the conventional radiocarbon age (CRA), ${ }^{13} \mathrm{C}$ value (estimated and measured), calendar age at $2 \mathrm{SD}$ (cal. BP), sample material/ species, excavation context and sample weight.

\section{Sample pretreatment}

\section{Charcoal}

Clay, rootlets, shell fragments and other adhering non-charcoal materials were removed with tweezers from excavated charcoals before samples were submitted. Dating laboratories also physically removed possible contaminants before samples were washed in distilled water and crushed, or chopped to increase the surface area for subsequent pretreatment. Chemical pretreatment at the QDRC involved washing the charcoal in $10 \% \mathrm{HCl}$, whereas Waikato and Beta Analytic Inc. used the ABA method, in which the sample is heated with dilute $\mathrm{HCl}$ followed by dilute $\mathrm{NaOH}$, and given a final treatment in dilute hot $\mathrm{HCl}$. Identification of wood charcoals used in radiocarbon dating is routinely carried out in New Zealand, and at some contract archaeology companies working in the Pacific, such as the International Archaeological Research Institute, Inc. (IARII). Charcoal from twigs or shortlived taxa is preferred for radiocarbon dating since it is unlikely to incorporate a high 'inbuilt' age (Anderson 1991). 
Table 17. Radiocarbon results from EPF archaeological investigations.

\begin{tabular}{|c|c|c|c|c|c|c|c|}
\hline Lab. Number & $\begin{array}{l}\text { Conventional Age } \\
\text { (BP) }\end{array}$ & $\begin{array}{l}\text { Calibrated Age } \\
\text { BP (2 SD) }\end{array}$ & $\Delta 13 C$ & Sample type & Species & $\begin{array}{l}\text { Unit and Depth } \\
\text { below surface }(\mathrm{cm})\end{array}$ & $\begin{array}{l}\text { Total weight } \\
\text { (grams) }\end{array}$ \\
\hline \multicolumn{8}{|l|}{ Ugaga } \\
\hline ANU-10774 & $1720 \pm 70$ & $1150-1390$ & $0.0 \pm 2.0 \mathrm{E}$ & Marine shell & Tridacna maxima & Sq. 15: 20-30 & 81.0 \\
\hline ANU-10776 & $1900 \pm 60$ & $1310-1590$ & $0.0 \pm 2.0 \mathrm{E}$ & Marine shell & Turbo argyrostromus & Sq. M8: 20-30 & 111.9 \\
\hline Beta-107951 & $2130 \pm 50$ & $1580-1850$ & 2.0 & Marine shell & Tridacna maxima & Sq. $-A 13: 30--40$ & 117.8 \\
\hline ANU-10772 & $2140 \pm 70$ & 1550-1890 & $0.0 \pm 2.0 \mathrm{E}$ & Marine shell & Trochus niloticus & Sq. 15:30-40 & 443.3 \\
\hline ANU-10773 & $2490 \pm 70$ & $1970-2320$ & $0.0 \pm 2.0 \mathrm{E}$ & Marine shell & Tridacna maxima & Sq. 09: 30-40 & 206.6 \\
\hline ANU-10777 & $2530 \pm 70$ & $2000-2340$ & $0.0 \pm 2.0 \mathrm{E}$ & Marine shell & Tridacna maxima & Sq. J9: $40-50$ & 137.0 \\
\hline ANU-10778 & $2600 \pm 70$ & $2090-2470$ & $0.0 \pm 2.0 \mathrm{E}$ & Marine shell & Tridacna maxima & Sq. T/U-1: 40-50 & 131.9 \\
\hline ANU-10775 & $2620 \pm 60$ & $2130-2470$ & $0.0 \pm 2.0 \mathrm{E}$ & Marine shell & Tridacna maxima & Sq. 19: 30-40 & 180.3 \\
\hline Beta-107952 & $2690 \pm 60$ & $2260-2650$ & 2.0 & Marine shell & Tridacna maxima & Sq. P10: $30-40$ & 132.7 \\
\hline Beta-107953 & $3150 \pm 70$ & $2760-3140$ & 3.2 & Marine shell & Trochus niloticus & Sq. C12: $50-60$ & 278.2 \\
\hline ANU-10734 & $97.7 \pm 0.8 \% \mathrm{M}$ & Modern & $-24 \pm 2.0 \mathrm{E}$ & Charcoal & - & Sq. 19: 20-30 & 100.0 \\
\hline Wk-5553 & $98.5 \pm 0.7 \% \mathrm{M}$ & Modern & $-27.7 \pm 0.2$ & Charcoal & - & Sq. K5: 30-40 & 18.2 \\
\hline Wk-5554 & $98.3 \pm 0.5 \% \mathrm{M}$ & Modern & $-26.9 \pm 0.2$ & Charcoal & - & Sq. J9: 20-30 & 11.0 \\
\hline Wk-5555 & $98.8 \pm 0.5 \% \mathrm{M}$ & Modern & $-26.8 \pm 0.2$ & Charcoal & - & Sq. K7: 20-30 & 100.0 \\
\hline Wk-5556 & $98.2 \pm 0.5 \% \mathrm{M}$ & Modern & $-25.3 \pm 0.2$ & Charcoal & - & Sq. P8: 20-30 & 56.1 \\
\hline \multicolumn{8}{|l|}{ Kulu } \\
\hline Beta 107947 & $2590 \pm 50$ & $2120-2380$ & 1.8 & Marine shell & Tridacna sp. & C11: 60-70 & - \\
\hline Beta 107948 & $2590 \pm 50$ & $2120-2380$ & 1.5 & Marine shell & Cerithium sp. & C11: $130-140$ & - \\
\hline Beta 107949 & $180 \pm 40$ & $0-280$ & -29.0 & Charcoal & Candle nut & C11:110-120 & - \\
\hline Beta 107950 & $220 \pm 50$ & $0-310$ & -24.0 & Charcoal & Candle nut & C11: 140-150 & - \\
\hline ANU-10727 & $820 \pm 100$ & 560-910 & $-24 \pm 2.0 \mathrm{E}$ & Charcoal & - & C9: 40-50 & 3.0 \\
\hline ANU-10743* & $117.2 \pm 2.7 \% \mathrm{M}$ & Modern & $-24 \pm 2.0 E$ & Charcoal & - & C11: 100-110 & 0.32 \\
\hline \multicolumn{8}{|l|}{ Karobo } \\
\hline ANU-10781 & $300 \pm 70$ & $0-500$ & $-24 \pm 2.0 E$ & Charcoal & - & Square Y, L3: 33 & 9.5 \\
\hline ANU-10780 & $1780 \pm 80$ & $1420-1830$ & $-24 \pm 2.0 \mathrm{E}$ & Charcoal & - & Square Ab-1, L7: 122 & 7.3 \\
\hline ANU-11067 & $1680 \pm 70$ & $1370-1700$ & $-24 \pm 2.0 \mathrm{E}$ & Charcoal & - & Square A2, L5: 100 & 6.2 \\
\hline ANU-11068 & $2130 \pm 120$ & $1740-2340$ & $-24 \pm 2.0 E$ & Wood & Palm cf. Cocos nucifora & Square A2, L5: 137 & 15.9 \\
\hline \multicolumn{8}{|l|}{ Malaqereqere } \\
\hline ANU-10453 & $830 \pm 90$ & $560-910$ & $-24 \pm 2.0 \mathrm{E}$ & Charcoal & - & Sq. A2: $60-70$ & 5.5 \\
\hline ANU-10452 & $460 \pm 60$ & $330-420$ & $-24 \pm 2.0 \mathrm{E}$ & Charcoal & - & Sq. A1: 10-20 & 78.3 \\
\hline ANU-10454 & $670 \pm 70$ & $520-680$ & $-24 \pm 2.0 E$ & Charcoal & - & Sq. A1: 40-50 & 11.0 \\
\hline \multicolumn{8}{|l|}{ Tuvu } \\
\hline ANU-11020 & $1570 \pm 100$ & $1260-1700$ & $-24 \pm 2.0 \mathrm{E}$ & Charcoal & - & TP 1, Spit IV & 3.0 \\
\hline \multicolumn{8}{|l|}{ Volivoli II } \\
\hline ANU-10449 & $1120 \pm 70$ & $810-1170$ & $-24 \pm 2.0 \mathrm{E}$ & Charcoal & - & TR. 1: 20-30 & 5.6 \\
\hline ANU-10450 & $1960 \pm 70$ & $1630-2040$ & $-24 \pm 2.0 \mathrm{E}$ & Charcoal & - & TR. 1: $40-50$ & 11.5 \\
\hline ANU-10451 & $1080 \pm 190$ & $580-1300$ & $-24 \pm 2.0 \mathrm{E}$ & Charcoal & - & TR.1: 50-60 & 4.6 \\
\hline \multicolumn{8}{|l|}{ Volivoli III } \\
\hline ANU-11018 & $290 \pm 60$ & $0-490$ & $-24 \pm 2.0 E$ & Charcoal & - & TP. 1, Spit 2 & 7.0 \\
\hline ANU-11016 & $1100 \pm 90$ & $770-1170$ & $-24 \pm 2.0 \mathrm{E}$ & Charcoal & - & TP. 1, Spit 5 & 2.2 \\
\hline ANU-11019 & $1060 \pm 80$ & $740-1070$ & $-24 \pm 2.0 \mathrm{E}$ & Charcoal & - & TP. 1, Spit 8 & 4.5 \\
\hline \multicolumn{8}{|l|}{ Qaranioso 1} \\
\hline ANU-11015 & $1280 \pm 120$ & $920-1350$ & $-24 \pm 2.0 \mathrm{E}$ & Charcoal & - & TP. 1: 30 & - \\
\hline
\end{tabular}


Table 17 continued

\begin{tabular}{|c|c|c|c|c|c|c|c|}
\hline Lab. Number & $\begin{array}{l}\text { Conventional Age } \\
\text { (BP) }\end{array}$ & $\begin{array}{l}\text { Calibrated Age } \\
\text { BP (2 SD) }\end{array}$ & ${ }^{\Delta 13} \mathrm{C}$ & Sample type & Species & $\begin{array}{l}\text { Unit and Depth } \\
\text { below surface }(\mathrm{cm})\end{array}$ & $\begin{array}{l}\text { Total weight } \\
\text { (grams) }\end{array}$ \\
\hline \multicolumn{8}{|l|}{ Qaranioso II } \\
\hline ANU-11014 & $660 \pm 60$ & $530-670$ & $-24 \pm 2.0 \mathrm{E}$ & Charcoal & - & TP. 1: 30 & - \\
\hline \multicolumn{8}{|l|}{ Natunuku } \\
\hline ANU-10382 & $98.4 \pm 1.0 \% \mathrm{M}$ & Modern & $-24 \pm 2.0 \mathrm{E}$ & Charcoal & - & Tr.3, Sq.6: 25-40 & 7.0 \\
\hline ANU-10699 & $1160 \pm 70$ & $600-890$ & $0.0 \pm 2.0 \mathrm{E}$ & Marine shell & Tridacna sp. & Tr.3, Sq.A5: 30-40 & 65.9 \\
\hline ANU-10381 & $99.7 \pm 0.8 \% \mathrm{M}$ & Modern & $-24 \pm 2.0 \mathrm{E}$ & Charcoal & - & Tr.3, Sq.A5: 10-20 & 20.4 \\
\hline ANU-10700 & $380 \pm 70$ & $160-230$ & $0.0 \pm 2.0 \mathrm{E}$ & Marine shell & Trochus sp. & Tr.3, Sq.A5: 10-20 & 172.8 \\
\hline ANU-10698 & $2780 \pm 90$ & $2310-2720$ & $0.0 \pm 2.0 \mathrm{E}$ & Marine shell & Tridacna sp. & Tr.3, Sq.A5: 20-30 & 95.8 \\
\hline ANU-11307 & $2600 \pm 60$ & $2110-2440$ & $0.0 \pm 2.0 \mathrm{E}$ & Marine shell & Species? & Tr. 3, no context & - \\
\hline ANU-11306 & $1170 \pm 50$ & $630-840$ & $0.0 \pm 2.0 \mathrm{E}$ & Marine shell & Species? & Tr. 3, no context & - \\
\hline ANU-11305 & $2900 \pm 50$ & $2490-2770$ & $0.0 \pm 2.0 \mathrm{E}$ & Marine shell & Species? & Tr. 3, no context & - \\
\hline \multicolumn{8}{|l|}{ Navatu 17A } \\
\hline ANU-10385 & $350 \pm 70$ & $150-500$ & $-24 \pm 2.0 \mathrm{E}$ & Charcoal & - & Tr. A1, L1: 100 & 40.7 \\
\hline ANU-10388 & $104.0 \pm 1.1 \% \mathrm{M}$ & Modern & $-24 \pm 2.0 \mathrm{E}$ & Charcoal & - & Tr. B2, L1: 20 & 5.0 \\
\hline ANU-10710 & $720 \pm 70$ & $250-500$ & $0.0 \pm 2.0 \mathrm{E}$ & Marine shell & Anadara sp. & Tr. B2, L1: 50 & 116.8 \\
\hline ANU-10389 & $330 \pm 60$ & $150-490$ & $-24 \pm 2.0 \mathrm{E}$ & Charcoal & - & Tr. B1, L1: 86 & 20.7 \\
\hline ANU-10709 & $980 \pm 70$ & $480-670$ & $0.0 \pm 2.0 \mathrm{E}$ & Marine shell & Gafrarium sp. & Tr. B1, L2: 120 & 51.7 \\
\hline ANU-10384 & $870 \pm 70$ & $660-910$ & $-24 \pm 2.0 \mathrm{E}$ & Charcoal & - & Tr. B1, L2: 140 & 6.0 \\
\hline ANU-10387 & $1240 \pm 140$ & $800-1350$ & $-24 \pm 2.0 \mathrm{E}$ & Charcoal & - & Tr. B1+2, L4: 215 & 2.4 \\
\hline ANU-10390 & $1010 \pm 140$ & $660-1180$ & $-24 \pm 2.0 \mathrm{E}$ & Charcoal & - & Tr. B1+2, L4: 220 & 2.1 \\
\hline ANU-10386 & $1670 \pm 70$ & $1360-1690$ & $-24 \pm 2.0 \mathrm{E}$ & Charcoal & - & Tr. B3+4, L4: 230 & 7.3 \\
\hline ANU-10708 & $1980 \pm 70$ & $1360-1710$ & $0.0 \pm 2.0 \mathrm{E}$ & Marine shell & Tridacna sp. & Tr. B3+4, L4: 230 & 78.8 \\
\hline \multicolumn{8}{|l|}{ Votua } \\
\hline Wk-5366 & $2970 \pm 50$ & $2620-2870$ & $-0.3 \pm 0.2$ & Marine shell & Anadara antiquata & Testpit 1, 60-70 & 133.3 \\
\hline Wk-5367 & $2930 \pm 50$ & $2540-2830$ & $-0.2 \pm 0.2$ & Marine shell & Anadara antiquata & Testpit 1, 30-40 & 198.8 \\
\hline ANU-10706 & $2520 \pm 120$ & $2170-2790$ & $-24 \pm 2.0 \mathrm{E}$ & Charcoal & - & Testpit 1, 40-50 & 7.1 \\
\hline Wk-5368 & $2940 \pm 50$ & $2570-2840$ & $-0.4 \pm 0.2$ & Marine shell & Anadara antiquata & Area 1:20-30 & 157.7 \\
\hline Wk-5369 & $2950 \pm 50$ & $2600-2850$ & $-0.2 \pm 0.2$ & Marine shell & Anadara antiquata & Area 1: 10-20 & 183.4 \\
\hline ANU-10707 & $2670 \pm 70$ & $2460-2880$ & $-24 \pm 2.0 \mathrm{E}$ & Charcoal & - & Area 1:20-30 & 4.6 \\
\hline ANU-11069 A & $2490 \pm 60$ & $1990-2300$ & $0.0 \pm 2.0 \mathrm{E}$ & Marine shell & Trochus niloticus & Area 1:20-30 & 68.0 \\
\hline ANU-11069 B & $2990 \pm 60$ & $2650-2930$ & $0.0 \pm 2.0 \mathrm{E}$ & Marine shell & Trochus niloticus & Area 1: 10-20 & 40.0 \\
\hline ANU-11528 & $2680 \pm 70$ & $2470-2920$ & $-26.4 \pm 0.2$ & Charcoal & - & Area 2, Sq. $4: 60$ & 8.1 \\
\hline ANU-11527 & $2850 \pm 50$ & $2440-2730$ & $-1.5 \pm 0.1$ & Marine shell & Anadara antiquata & Area 2, Sq. 1:60-70 & 43.6 \\
\hline \multicolumn{8}{|l|}{ Sovanibeka } \\
\hline 0ZF882* & $840 \pm 40$ & $670-770$ & -20.0 & Bone & Rattus praetor & Test Pit 1:50-60 & 1.7 \\
\hline ANU-10779 & $2820 \pm 70$ & $2360-2720$ & $0.0 \pm 0.2 \mathrm{E}$ & Marine shell & Anadara antiquata & Test Pit 2: 30-50 & 7.3 \\
\hline ANU-11246 & $4290 \pm 60$ & $4540-4960$ & $-2.5 \pm 0.2$ & Land snail & Gonatorhaphe lavens & Test Pit 2: 55-58 & 34.1 \\
\hline
\end{tabular}

* = AMS determination.

' $\mathrm{E}$ ' in the delta ${ }^{13} \mathrm{C}$ column indicates the use of an estimated ${ }^{13} \mathrm{C}$ value.

Table 18. Navatu 17A thermoluminescence results (Trench B, Square 1).

\begin{tabular}{lllllll}
\hline TL No. & Unit/Depth (cm) & Equivalent dose (Gy) & a-value & Total dose rate (Gy ka-1) & Age & Age range \\
\hline 98001 & Tr. B, Sq. 1: 140 & $1.59 \pm 0.3$ & $0.22 \pm 0.03$ & $1.22 \pm 0.11$ & $1300 \pm 275$ & $1575-1025$ \\
98002 & Tr. B, Sq. 1: 230 & $2.44 \pm 0.3$ & $0.28 \pm 0.03$ & $1.10 \pm 0.13$ & $2230 \pm 380$ & $2610-1850$ \\
\hline
\end{tabular}




\section{cal. years BP}

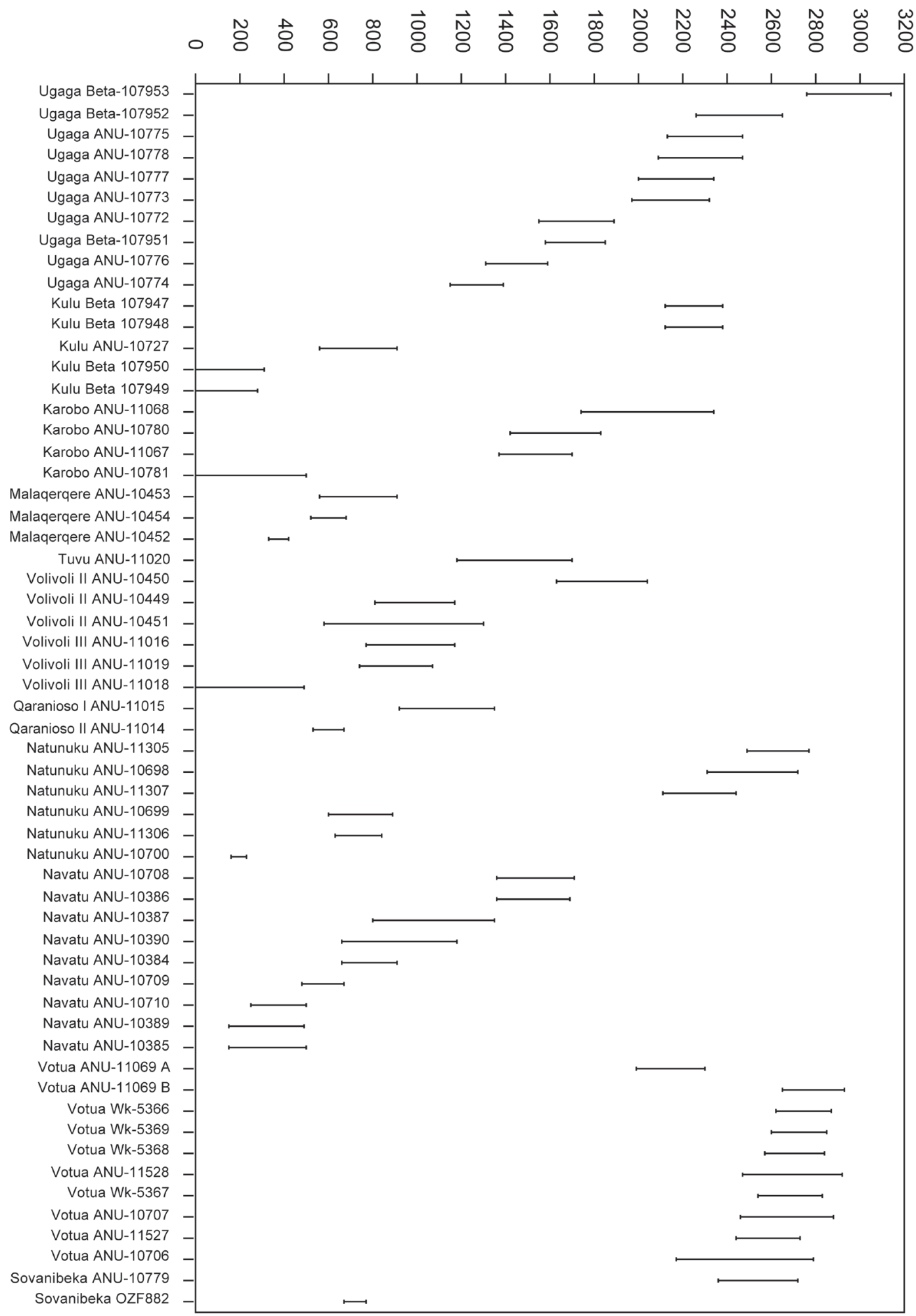

Figure 83. EPF calibrated age determinations at 2SD. 
Radiocarbon laboratories do not currently identify archaeological charcoals from the tropical Pacific. Consequently, ages on charcoal from Fiji and from elsewhere in the West and Central Pacific may be older than the cultural activity associated with the sample.

\section{Palm wood}

The surfaces of a palm-wood sample dated at the QDRC were scraped clean and the wood was chopped into small splinters and milled. The sample was washed in hot $10 \% \mathrm{HCl}$, rinsed and dried. The sample was soxlet extracted with ethanol, ethanol/chloroform (2:1) and water, bleached with sodium perchlorate, rinsed and dried.

\section{Marine and terrestrial shell}

Marine-shell samples were identified using a reference collection at the ANU, and cleaned with a dental drill (QDRC) or acid etched (Beta Analytic Inc., Waikato). The Waikato laboratory tests marine shells for recrystallisation before dating, but this procedure was not standard for marine shell dated at the QDRC and Beta Analytic Inc. Marine shells from the Votua site were examined for recrystallisation with X-ray diffraction, which identified the samples as $100 \%$ aragonite, indicating that the prehistoric shell samples were not affected by the transformation of biogenic aragonite to calcite. Land snails were identified by Winston Ponder (Australian Museum), with samples of a single species sorted and pretreated by gently crushing the shell and repeatedly washing the residue in an ultrasonic bath filled with distilled water until adhering cave sediments had been removed from the surface shell.

\section{Bone}

Macroscopic contaminants attached to the bone sample were removed with tweezers at ANSTO, the dry bone was crushed in a mortar and pestle, and the fragments were washed in an ultrasonic bath filled with Milli-RO water until clean. The fragments were placed in a freeze dryer for one to two days, then ground to powder with a particle size of less than $200 \mu \mathrm{m}$. Chemical pretreatment involved removal of the inorganic bone component with an acid-alkali wash and gelatin extraction in heated $0.01 \mathrm{M} \mathrm{HCl}$, followed by separation of the collagen in solution with a centrifuge, and two to three days of freeze drying to dry the gelatin.

\section{CRA and calibration}

Isotopic fractionation refers to the difference in the proportion of ${ }^{14} \mathrm{C}$ to ${ }^{12} \mathrm{C}$ in a living organism or sample, and the proportion of ${ }^{14} \mathrm{C}$ to ${ }^{12} \mathrm{C}$ in the carbon reservoir. The conventional radiocarbon age (CRA) is corrected for fractionation by measuring the delta ${ }^{13} \mathrm{C}$ of a sample with a mass spectrometer, or by using an estimated value for marine and terrestrial carbon reservoirs. Some CRA ages from the QDRC had measured delta ${ }^{13} \mathrm{C}$ values, but most incorporated estimated values of $-24 \pm 2.0 \%$ o for charcoal and $0 \pm 2.0 \%$ o for marine shell relative to VDPB. For Fijian charcoal samples the measured delta ${ }^{13} \mathrm{C}$ varied from $-29.0 \%$ to $-24.0 \%$ (delta ${ }^{13} \mathrm{C}$ range $=5.0 \%$ ). The majority of marine shells had a similar range of $5.7 \%$ o (3.2\% to $-2.5 \%$ ), suggesting that in most cases the use of estimated delta ${ }^{13} \mathrm{C}$ values would not have made a significant change to the CRA. However, a Trachycardium shell from the Yanuca site on Viti Levu was measured at $-12.3 \%$ (Clark and Anderson 2001b), indicating that some marine shellfish from locations adjacent to freshwater sources can have $\operatorname{delta}^{13} \mathrm{C}$ values significantly different from the estimated marine reservoir value. CRAs and standard errors (1SD) were rounded to the nearest decade.

Calibrations were by CALIB 5.0 (Stuiver and Reimer 1993), with terrestrial samples calculated with the Southern Hemisphere curve (McCormac et al. 2004), marine samples 
with the Marine curve of Hughen et al. (2004), and mixed terrestrial-marine samples with the Southern Hemisphere combined curves of Hughen et al. (2004) and McCormac et al. (2004). DeltaR was set at 0 for marine samples. DeltaR values of +25 years (Petchey 1995) and $+45 \pm 30$ years (Stuiver and Braziunas 1993) have been used to calibrate radiocarbon determinations from Fiji, and a marine reservoir value of $38 \pm 16$ years has been calculated for Viti Levu on coral rings (Toggweiler et al. 1991). In the absence of marine-reservoir values for specific locations, we prefer to use the 0 value, given the diversity of marine and coastal environments in Fiji. Marineshell-charcoal pairs from archaeological sites in Viti Levu and the Lau Group (Clark 2000; Thomas et al. 2004) also suggest that the value of DeltaR is relatively small. Samples of human bone (Table 19) were calibrated with the terrestrial-marine Southern Hemisphere calibration curve, with DeltaR set at $50 \%$ because of the likelihood of marine carbon in the diet (Leach et al. 2001). Calibrated results are reported at two standard deviations and rounded to the nearest decade.

\section{Ugaga Island}

Four charcoal samples were sent to the Waikato Radiocarbon Dating Laboratory and one to the QDRC. Modern results were obtained, necessitating a new sample submission strategy (Wk-5553, Wk-5554, Wk-5555, Wk-5556, ANU-10734). Graphing the weight of charcoal recovered from each square and spit at the site revealed that the samples came from squares with abundant charcoal that was probably introduced by recent ovens dug into the site. Because of the difficulty of identifying prehistoric charcoal samples in the mixed stratigraphy at Ugaga, marine shells were selected for radiocarbon dating instead of charcoal.

Two factors supported the use of marine shell for dating the site. First, large and heavy shells are less susceptible to profile migration through the digging activities of crabs, people and other agents. Second, local informants said shellfish were not plentiful on the island's small reef, and had not been collected from Ugaga in recent times. This suggested much of the marine shell on Ugaga could have been deposited in prehistoric times. Ten marine-shell samples were dated, three at Beta Analytic, Inc. and seven at the QDRC.

In contrast to the charcoal results, all of the marine-shell samples gave prehistoric ages, with CRA results ranging from 1720-3150 BP. Five of the calibrated dates lay between 1970 and $2650 \mathrm{cal}$. BP (ANU-10773, ANU-10777, ANU-107778, ANU-10775, Beta-107952), and four between 1150 and $1890 \mathrm{cal}$. BP (ANU-10774, ANU-10766, Beta-107951, ANU-10772). A large unbroken Trochus niloticus shell from Square D12 had an age of 2760-3140 cal. BP (Beta-107953). The sample was collected from a grey sand at $60 \mathrm{~cm}$ depth that contained a few plain sherds and several large fragments of coral.

Considering the amount of disturbance at Ugaga (Chapter 5), there is a reasonable age-depth relationship over the site, with the most recent shell determinations from the $20-30 \mathrm{~cm}$ spit, and the oldest ${ }^{14} \mathrm{C}$ dates from lower spits. Square $\mathrm{I} 5$ had stratigraphically consistent dates from upper and lower spits (ANU-10774, ANU-10772). Modern determinations (ANU-10734, Wk-5554) on charcoal from squares I9 $(20-30 \mathrm{~cm})$ and $\mathrm{J} 9(20-30 \mathrm{~cm})$ were contradicted by much older ages on shell from the same squares (ANU-10775, ANU-10775), emphasising the capacity for earth ovens (lovo) to introduce large amounts of modern charcoal into prehistoric levels.

Radiocarbon dates from Ugaga show the small island was used in the Lapita era and for the following two millennia, with only limited evidence in the ceramics and radiocarbon results for human use in the past 1000 years. 
Table 19. An inventory of radiocarbon results from archaeological sites in Fiji.

\begin{tabular}{|c|c|c|c|c|c|c|c|c|c|c|}
\hline Island & Site & Lab Code & CRA & CRA SE & C13 & Cal. BP & $\begin{array}{l}\text { Sample } \\
\text { Type }\end{array}$ & Identification & Context & Reference \\
\hline Aiwa Lailai & DR:1 & Beta-172192 & 1510 & 40 & - & $1290-1410$ & Bone & Pteropus sp. & $\mathrm{lllb} / 4$ & O'Day et al. (2007) \\
\hline Aiwa Lailai & $\mathrm{DR}: 2$ & Beta-172191 & 2300 & 50 & - & $2140-2350$ & Bone & Gallus galllus & $\mathrm{IV} / 5$ & 0'Day et al. (2007) \\
\hline Aiwa Levu & AC2:1 & Beta-165465 & 2380 & 40 & - & $2160-2490$ & Bone & Gallus galllus & $11 / 2$ & O'Day et al. (2007) \\
\hline Aiwa Levu & $\mathrm{AC} 2: 2$ & Beta-165466 & 960 & 40 & - & $750-920$ & Bone & Pteropus sp. & 1/1 & O'Day et al. (2007) \\
\hline Aiwa Levu & $A C 2: 3$ & Beta-165467 & 1630 & 40 & - & $1370-1550$ & Bone & Pteropus sp. & $11 / 2$ & O'Day et al. (2007) \\
\hline Aiwa Levu & AR1:2 & Beta-164251 & 280 & 40 & - & $150-450$ & Bone & Pteropus sp. & 1/1 & O'Day et al. (2007) \\
\hline Aiwa Levu & AR1:2 & Beta-164252 & 2310 & 40 & - & $1970-2290$ & Bone & Human & III/13 & 0'Day et al. (2007) \\
\hline Aiwa Levu & AR1:2 & Beta- 164260 & 200 & 40 & - & $0-300$ & Charcoal & - & $11 / 5$ & 0'Day et al. (2007) \\
\hline Aiwa Levu & AR1:2 & Beta-164261 & 570 & 40 & - & $500-630$ & Charcoal & - & III/10 & O'Day et al. (2007) \\
\hline Aiwa Levu & AR1:4 & Beta-164258 & 360 & 40 & - & $310-490$ & Bone & Pteropus sp. & $11 / 3$ & O'Day et al. (2007) \\
\hline Aiwa Levu & GR1 & Beta-165469 & 370 & 40 & - & $310-490$ & Bone & Ducula sp. & $111 / 5$ & O'Day et al. (2007) \\
\hline Beqa & Kulu & ANU-10727 & 820 & 100 & $-24 \pm 2.0 \mathrm{E}$ & $560-910$ & Marine shell & - & C9: 40-50 cmbs & ANU-EPF \\
\hline Beqa & Kulu & Beta 107947 & 2590 & 50 & 1.8 & $2120-2380$ & Marine shell & Tridacna sp. & $\mathrm{C} 11,60-70 \mathrm{cmbs}$ & ANU-EPF \\
\hline Beqa & Kulu & Beta 107948 & 2590 & 50 & 1.5 & $2120-2380$ & Marine shell & Cerithium sp. & $\mathrm{C} 11,130-140 \mathrm{cmbs}$ & ANU-EPF \\
\hline Beqa & Kulu & Beta 107949 & 180 & 40 & -29.0 & $0-280$ & Charcoal & Candle nut & $\mathrm{C} 11,110-120 \mathrm{cmbs}$ & ANU-EPF \\
\hline Beqa & Kulu & Beta 107950 & 220 & 50 & -24.0 & $0-310$ & Charcoal & Candle nut & $\mathrm{C} 11,140-150 \mathrm{cmbs}$ & ANU-EPF \\
\hline Beqa & Nacuromoce & $?$ & 626 & 60 & - & $104-430$ & Marine shell & Trochus niloticus & Layer 1 & Crosby (1988: Appendix) \\
\hline Beqa & Rukua & $?$ & 1670 & 60 & - & $1370-1690$ & Charcoal & - & Layer 3 & Crosby (1988: Appendix) \\
\hline Beqa & Rukua & $?$ & 1676 & 60 & - & $1090-1340$ & Marine shell & Atactodea striata & Layer 3 & Crosby (1988: Appendix) \\
\hline Beqa & Kulu & ANU-10743* & $117.2 \pm 2.7 \% \mathrm{M}$ & - & $-24 \pm 2.0 \mathrm{E}$ & - & Chacoal & - & C11: 100-110 & ANU-EPF \\
\hline Beqa & Natunuku & ANU-10382 & $98.4 \pm 1.0 \% \mathrm{M}$ & - & $-24 \pm 2.0 \mathrm{E}$ & - & Charcoal & - & Tr.3, Sq.6: 25-40 & ANU-EPF \\
\hline Lakeba & Kedeke & NZ-4042 & 690 & 50 & - & $550-670$ & Charcoal & - & Sq A, Layer J7 & Best (1984: 146) \\
\hline Lakeba & Kedeke & $N Z-4043$ & 110 & 60 & - & $0-280$ & Charcoal & - & Sq D, Layer 2 & Best (1984: 146) \\
\hline Lakeba & Laselase & $N Z-4039$ & 80 & 70 & - & - & Charcoal & - & B.E & Best (1984: 87) \\
\hline Lakeba & Laselase & $N Z-4040$ & 2000 & 100 & - & $1620-2150$ & Charcoal & - & $\mathrm{L}, \mathrm{M}$ & Best (1984: 87) \\
\hline Lakeba & Laselase & NZ-4041 & 2280 & 100 & - & $1950-2490$ & Charcoal & - & S-U & Best (1984: 87) \\
\hline Lakeba & Laselase & NZ-4903 & 1515 & 60 & - & $1280-1520$ & Charcoal & - & $J 2$ & Best (1984: 87) \\
\hline Lakeba & Laselase & NZ-5182 & 1200 & 40 & - & $660-850$ & Marine shell & Turbo chrysostomus & $\mathrm{J} 1$ & Best (1984: 87) \\
\hline Lakeba & Qaranipuqa & NZ-4588 & 1790 & 40 & - & $1260-1430$ & Marine shell & Tridacna maxima & Layer F3 & Best (1984: 75) \\
\hline Lakeba & Qaranipuqa & NZ-4589 & 2600 & 50 & - & $2130-2400$ & Marine shell & Lambis lambis & Layer T & Best (1984: 75) \\
\hline Lakeba & Qaranipuqa & NZ-4590 & 2830 & 60 & - & $2390-2730$ & Marine shell & Conus leopardus & LayerW & Best (1984: 75) \\
\hline Lakeba & Qaranipuqa & NZ-4591 & 2230 & 50 & - & $1700-1960$ & Marine shell & Turbo bruneus & Layer K4 & Best (1984: 75) \\
\hline Lakeba & Qaranipuqa & NZ-4592 & 1770 & 90 & - & $1410-1860$ & Charcoal & - & Layer F3 & Best (1984: 75) \\
\hline Lakeba & Qaranipuqa & NZ-4593 & 2110 & 90 & - & $1820-2310$ & Charcoal & - & Layer K1 & Best (1984: 75) \\
\hline Lakeba & Qaranipuqa & NZ-4594 & 2960 & 70 & - & $2860-3320$ & Charcoal & - & Layer N & Best (1984: 75) \\
\hline Lakeba & Qaranipuqa & NZ-4595 & 1120 & 90 & - & $780-1230$ & Charcoal & Coconut & Layer W & Best (1984: 75) \\
\hline Lakeba & Qaranipuqa & NZ-4596 & 2620 & 100 & - & $2360-2850$ & Charcoal & - & Layer T & Best (1984: 75) \\
\hline
\end{tabular}


Table 19 continued

\begin{tabular}{|c|c|c|c|c|c|c|c|c|c|c|}
\hline Island & Site & Lab Code & CRA & CRA SE & C13 & Cal. BP & $\begin{array}{l}\text { Sample } \\
\text { Type }\end{array}$ & Identification & Context & Reference \\
\hline Lakeba & Qaranipuqa & NZ-4808 & 2260 & 80 & - & $2000-2350$ & Charcoal & - & Layer N & Best (1984: 75) \\
\hline Lakeba & Qaranipuqa & NZ-4810 & 4010 & 620 & - & $2530-5580$ & Bone & Turtle & Layer W & Best (1984: 75) \\
\hline Lakeba & Qaranipuqa & NZ-4904 & 394 & 84 & - & $160-540$ & Charcoal & - & Layer A2 & Best (1984: 75) \\
\hline Lakeba & Qaranipuqa & NZ-4905 & 892 & 60 & - & $670-910$ & Charcoal & - & Layer E2 & Best (1984: 75) \\
\hline Lakeba & Qaranipuqa & NZ-4906 & 2960 & 160 & - & $2330-3130$ & Bone & Turtle & Layer W & Best (1984: 75) \\
\hline Lakeba & Ulunikoro & NZ-4044 & 1020 & 90 & - & $690-1060$ & Charcoal & - & Sq 3, Layer B & Best (1984: 129) \\
\hline Lakeba & Ulunikoro & NZ-4581 & 930 & 40 & - & $480-620$ & Marine shell & Turbo chrysostomus & Sq 15, Layer B2 & Best (1984: 129) \\
\hline Lakeba & Ulunikoro & NZ-4582 & 970 & 40 & - & $500-640$ & Marine shell & Turbo chrysostomus & Sq 17, Layer B3 & Best (1984: 129) \\
\hline Lakeba & Ulunikoro & NZ-4583 & 980 & 40 & - & $510-640$ & Marine shell & Turbo chrysostomus & Sq 12, Layer B1 & Best (1984: 129) \\
\hline Lakeba & Ulunikoro & NZ-4584 & 340 & 50 & - & $290-490$ & Charcoal & - & Sq 8, Layer B5 & Best (1984: 129) \\
\hline Lakeba & Ulunikoro & NZ-4585 & 900 & 90 & - & $650-950$ & Charcoal & - & Sq 15, Layer B3 & Best (1984: 129) \\
\hline Lakeba & Ulunikoro & NZ-4586 & 1010 & 90 & - & $690-1060$ & Charcoal & - & Sq 12/13, Layer B1 & Best (1984: 129) \\
\hline Lakeba & Ulunikoro & NZ-4587 & 1030 & 90 & - & $690-1070$ & Bone & Turtle & Sq 12, Layer B1 & Best (1984: 129) \\
\hline Lakeba & Ulunikoro & NZ-5179 & 984 & 50 & - & $500-650$ & Marine shell & Turbo crassus & Sq 12, Layer A & Best (1984: 129) \\
\hline Lakeba & Ulunikoro & NZ-5180 & 1315 & 40 & - & $760-940$ & Marine shell & Turbo chrysostomus & Sq 8, Layer B5 & Best (1984: 129) \\
\hline Lakeba & Ulunikoro & NZ-5181 & 411 & 40 & - & $0-130$ & Marine shell & Turbo chrysostomus & Sq 10, Layer B & Best (1984: 129) \\
\hline Lakeba & Wakea & NZ-4807 & 2701 & 120 & - & $2360-3060$ & Charcoal & - & $\begin{array}{l}\text { Sq 25, Layer B18, approx: } \\
225 \text { cmbs }\end{array}$ & Best (1984) \\
\hline Lakeba & Wakea & NZ-4809 & 2604 & 80 & - & $1050-2520$ & Marine shell & Trochus niloticus & $\begin{array}{l}\text { Sq 19, Layer B20a, approx: } \\
225 \text { cmbs }\end{array}$ & Best (1984) \\
\hline Mago & Sovanibeka & ANU-10779 & 2820 & 70 & $0.0 \pm 2.0 \mathrm{E}$ & $2360-2720$ & Marine shell & Anadara antiquata & Test Pit 2, $30-50 \mathrm{cmbs}$ & ANU-EPF \\
\hline Mago & Sovanibeka & ANU-11246 & 4290 & 60 & $-2.5 \pm 0.2$ & $4540-4960$ & Land snail & Gonatorhaphe lavens & Test Pit 2, 55-58 cmbs & ANU-EPF \\
\hline Mago & Sovanibeka & 0ZF882 & 840 & 40 & -20.0 & $670-770$ & Bone & Rattus praetor & Test Pit 1: $50-60 \mathrm{cmbs}$ & ANU-EPF \\
\hline Mago & Votua & ANU-10706 & 2520 & 120 & $-24 \pm 2.0 \mathrm{E}$ & $2170-2790$ & Charcoal & - & Testpit 1, 40-50 cmbs & ANU-EPF \\
\hline Mago & Votua & ANU-10707 & 2670 & 70 & $-24 \pm 2.0 \mathrm{E}$ & $2460-2880$ & Charcoal & - & Area $1,20-30 \mathrm{cmbs}$ & ANU-EPF \\
\hline Mago & Votua & ANU-11069 A & 2490 & 60 & $0.0 \pm 2.0 \mathrm{E}$ & $1990-2300$ & Marine shell & Trochus niloticus & Area $1,20-30 \mathrm{cmbs}$ & ANU-EPF \\
\hline Mago & Votua & ANU-11069 B & 2990 & 60 & $0.0 \pm 2.0 \mathrm{E}$ & $2650-2930$ & Marine shell & Trochus niloticus & Area $1,10-20 \mathrm{cmbs}$. & ANU-EPF \\
\hline Mago & Votua & ANU-11527 & 2850 & 50 & $-1.5 \pm 0.1$ & $2440-2730$ & Marine shell & Anadara antiquata & Area 2, Sq. 1, 60-70 cmbs & ANU-EPF \\
\hline Mago & Votua & ANU-11528 & 2680 & 70 & $-26.4 \pm 0.2$ & $2470-2920$ & Charcoal & - & Area 2, Sq. 4, $60 \mathrm{cmbs}$ & ANU-EPF \\
\hline Mago & Votua & Wk-5366 & 2970 & 50 & $-0.3 \pm 0.2$ & $2620-2870$ & Marine shell & Anadara antiquata & Testpit 1, 60-70 cmbs & ANU-EPF \\
\hline Mago & Votua & Wk-5367 & 2930 & 50 & $-0.2 \pm 0.2$ & $2540-2830$ & Marine shell & Anadara antiquata & Testpit $1,30-40 \mathrm{cmbs}$ & ANU-EPF \\
\hline Mago & Votua & Wk-5368 & 2940 & 50 & $-0.4 \pm 0.2$ & $2570-2840$ & Marine shell & Anadara antiquata & Area $1,20-30 \mathrm{cmbs}$ & ANU-EPF \\
\hline Mago & Votua & Wk-5369 & 2950 & 50 & $-0.2 \pm 0.2$ & $2600-2850$ & Marine shell & Anadara antiquata & Area $1,10-20 \mathrm{cmbs}$ & ANU-EPF \\
\hline Moturiki & Naitabale & NUTA2-5198a & 2492 & 70 & -15.4 & $2110-2650$ & Bone & Human & $\mathrm{T} 1: 150 \mathrm{cmbs}$ & P. Nunn, pers. comm., USP \\
\hline Moturiki & Naitabale & NUTA2-5198b & 2547 & 90 & -15.4 & $2160-2690$ & Bone & Human & $\mathrm{T} 1: 150 \mathrm{cmbs}$ & P. Nunn, pers. comm., USP \\
\hline Moturiki & Naitabale & NUTA2-5198c & 2550 & 80 & -15.4 & $2630-2690$ & Bone & Human & $\mathrm{T} 1: 150 \mathrm{cmbs}$ & P. Nunn, pers. comm., USP \\
\hline Moturiki & Naitabale & NUTA2-5200a & 2664 & 70 & -15.1 & $2360-2720$ & Bone & Human & $\mathrm{T} 1: 150 \mathrm{cmbs}$ & P. Nunn, pers. comm., USP \\
\hline Moturiki & Naitabale & NUTA2-5200b & 2637 & 70 & -15.1 & $2345-2710$ & Bone & Human & $\mathrm{T} 1: 150 \mathrm{cmbs}$ & P. Nunn, pers. comm., USP \\
\hline
\end{tabular}


Table 19 continued

\begin{tabular}{|c|c|c|c|c|c|c|c|c|c|c|}
\hline Island & Site & Lab Code & CRA & CRA SE & C13 & Cal. BP & $\begin{array}{l}\text { Sample } \\
\text { Type }\end{array}$ & Identification & Context & Reference \\
\hline Moturiki & Naitabale & NUTA2-5200C & 2576 & 80 & -15.1 & $2190-2710$ & Bone & Human & $\mathrm{T} 1: 150 \mathrm{cmbs}$ & P. Nunn, pers. comm., USP \\
\hline Moturiki & Naitabale & Wk-11474 & 339 & 40 & -27.2 & $300-470$ & Charcoal & - & $\mathrm{T} 1: 75 \mathrm{cmbs}$ & P. Nunn, pers. comm., USP \\
\hline Moturiki & Naitabale & Wk-11475 & 2438 & 50 & -27.4 & $2330-2700$ & Charcoal & - & $\mathrm{T} 1: 75 \mathrm{cmbs}$ & P. Nunn, pers. comm., USP \\
\hline Moturiki & Naitabale & Wk-11476 & 2650 & 40 & -26.8 & $2500-2790$ & Charcoal & - & $\mathrm{T} 1: 145 \mathrm{cmbs}$ & P. Nunn, pers. comm., USP \\
\hline Moturiki & Naitabale & Wk-11477 & 2519 & 40 & -25.6 & $2360-2710$ & Charcoal & - & P3: 85 cmbs & P. Nunn, pers. comm., USP \\
\hline Moturiki & Naitabale & Wk-11478 & 2644 & 40 & -26.3 & $2500-2780$ & Charcoal & - & P3: $115 \mathrm{cmbs}$ & P. Nunn, pers. comm., USP \\
\hline Moturiki & Naitabale & Wk-11479 & 295 & 40 & -27.4 & $2890-3210$ & Charcoal & - & $\mathrm{R} 2: 55 \mathrm{cmbs}$ & P. Nunn, pers. comm., USP \\
\hline Moturiki & Naitabale & Wk-11480 & 2576 & 40 & -24.4 & $2370-2750$ & Charcoal & - & $\mathrm{R} 2: 105 \mathrm{cmbs}$ & P. Nunn, pers. comm., USP \\
\hline Moturiki & Naitabale & Wk-11481 & 2854 & 50 & -28.0 & $2780-3060$ & Charcoal & - & $\mathrm{R} 2: 115 \mathrm{cmbs}$ & P. Nunn, pers. comm., USP \\
\hline Moturiki & Naitabale & Wk-11482 & 2456 & 40 & -28.4 & $2340-2700$ & Charcoal & - & $\mathrm{R} 2: 125 \mathrm{cmbs}$ & P. Nunn, pers. comm., USP \\
\hline Moturiki & Naitabale & Wk-13402 & 2974 & 40 & $2.1 \pm 0.2$ & $2680-2840$ & Marine shell & Anadara sp. & $\mathrm{T} 1: 150 \mathrm{cmbs}$ & P. Nunn, pers. comm., USP \\
\hline Moturiki & Naitabale & Wk-13403 & 2951 & 40 & $3.0 \pm 0.2$ & $2650-2840$ & Marine shell & Tectus pyramis & $\mathrm{T} 1: 150 \mathrm{cmbs}$ & P. Nunn, pers. comm., USP \\
\hline Moturiki & Naitabale & Wk-13404 & 2931 & 40 & $3.0 \pm 0.2$ & $2600-2840$ & Marine shell & Trochus niloticus & $\mathrm{T} 1: 150 \mathrm{cmbs}$ & P. Nunn, pers. comm., USP \\
\hline Moturiki & Naitabale & Wk-13405 & 2483 & 50 & -27.1 & $2350-2710$ & Charcoal & - & P3: $135 \mathrm{cmbs}$ & P. Nunn, pers. comm., USP \\
\hline Naigani & Naigani & NZ-5615 & 3142 & 50 & - & $2780-3080$ & Marine shell & Tridacna maxima & $\begin{array}{l}\text { Sq 7, extension, } 2.92 \mathrm{~m} \\
\text { below datum }\end{array}$ & Best (1981) \\
\hline Naigani & Naigani & NZ-5616 & 3152 & 50 & - & $2790-3100$ & Marine shell & Tridacna maxima & Sq $13,2.6 \mathrm{~m}$ below datum & Best (1981) \\
\hline Naigani & Naigani & NZ-5617 & 3052 & 50 & - & $2730-2950$ & Marine shell & Saccostrea cucullata & $\begin{array}{l}\text { Sq 7, extension, } 2.37 \mathrm{~m} \\
\text { below datum }\end{array}$ & Best (1981) \\
\hline Naigani & Naigani & NZ-5618 & 3082 & 70 & - & $2720-3050$ & Marine shell & Trochus niloticus & $\begin{array}{l}\text { Sq } 4 \text {, extension, } 1.72 \mathrm{~m} \\
\text { below datum }\end{array}$ & Best (1981) \\
\hline Viti Levu & Natunuku & ANU-10381 & $99.7 \pm 0.8 \% \mathrm{M}$ & - & $-24 \pm 2.0 \mathrm{E}$ & - & Charcoal & - & Tr.3, Sq.A5: 10-20 & ANU-EPF \\
\hline Viti Levu & Navatu & ANU-10388 & $104.0 \pm 1.1 \% \mathrm{M}$ & - & - & - & Charcoal & - & Tr. B2, L1: 20 & ANU-EPF \\
\hline Qoqo & Qoqo & Wk-16208 & 2925 & 40 & $3.2 \pm 0.2$ & $2570-2790$ & Marine shell & Trochus niloticus & F1: 188 & Nunn et al. (2006) \\
\hline Qoqo & Qoqo & Wk-16209 & 2990 & 40 & 0.0 & $2700-2850$ & Marine shell & $\begin{array}{l}\text { Anadara sp., Codakia } \\
\text { punctata, Gafrarium } \\
\text { tumidum }\end{array}$ & R2: 87 & Nunn et al. (2006) \\
\hline Qoqo & Qoqo & Wk-16218 & 2790 & 40 & -24.5 & $2760-2940$ & Charcoal & - & $\mathrm{R} 2: 118$ & Nunn et al. (2006) \\
\hline Qoqo & Qoqo & Wk-16219 & 402 & 40 & -24.0 & $330-500$ & Charcoal & - & P1: 185 & Nunn et al. (2006) \\
\hline Qoqo & Qoqo & Wk-16220 & 393 & 40 & -24.0 & $320-500$ & Charcoal & - & P1: 205 & Nunn et al. (2006) \\
\hline Taveuni & Navolivoli & GaK-2411 & 710 & 80 & - & $530-730$ & Charcoal & - & Structure \#11 & Frost (1970: 132) \\
\hline Taveuni & Navolivoli & GaK-2412 & 2050 & 150 & - & $1620-2330$ & Charcoal & - & $\begin{array}{l}\text { Trench \#14, } 30 \mathrm{~cm} \text { below } \\
\text { Layer A }\end{array}$ & Frost (1970: 137,141) \\
\hline Taveuni & Nawa & GaK-2414 & 710 & 80 & - & $530-730$ & Charcoal & - & Component $\mathrm{E}$ & Frost (1970: 113-114) \\
\hline Taveuni & Nayalayala & GaK-2507 & 340 & 80 & - & $0-510$ & Charcoal & - & Structure $\# 8,40 \mathrm{cmbs}$ & Frost (1970: 97) \\
\hline Taveuni & Qalau & GaK-2510 & 280 & 90 & - & $0-490$ & Charcoal & - & Structure \#5 & Frost (1970: 119) \\
\hline Taveuni & Taveuni & GaK-2413 & 740 & 70 & - & $550-740$ & Charcoal & - & Trench \#6, 40-70 cmbs & Frost (1970: 99,102) \\
\hline Taveuni & Taveuni & GaK-2508 & Modern & - & - & - & Charcoal & - & Trench \#2 & Frost (1970: 68) \\
\hline Taveuni & Taveuni & GaK-2509 & 620 & 90 & - & $470-720$ & Charcoal & - & Trench \#2, Layer A & Frost (1970: 76) \\
\hline Totoya & Lawaki Levu & Beta- 67623 & 2480 & 60 & - & $1980-2300$ & Marine shell & Tridacna sp. & TP-3, Layer III, $40 \mathrm{~cm} \mathrm{cmbs}$ & Clark and Cole (1997) \\
\hline
\end{tabular}


Table 19 continued

\begin{tabular}{|c|c|c|c|c|c|c|c|c|c|c|}
\hline Island & Site & Lab Code & CRA & CRA SE & C13 & Cal. BP & $\begin{array}{l}\text { Sample } \\
\text { Type }\end{array}$ & Identification & Context & Reference \\
\hline Totoya & Lawaki Levu & Beta- 67624 & 2370 & 60 & - & $1840-2150$ & Marine shell & Tectus sp. & TP-1, Layer II, $40 \mathrm{~cm}$ cmbs & Clark and Cole (1997) \\
\hline Ugaga & Ugaga & ANU-10772 & 2140 & 70 & $0.0 \pm 2.0 \mathrm{E}$ & $1550-1890$ & Marine shell & Trochus niloticus & I5: $30-40 \mathrm{cmbs}$ & ANU-EPF \\
\hline Ugaga & Ugaga & ANU-10773 & 2490 & 70 & $0.0 \pm 2.0 \mathrm{E}$ & $1970-2320$ & Marine shell & Tridacna maxima & 09: $30-40 \mathrm{cmbs}$ & ANU-EPF \\
\hline Ugaga & Ugaga & ANU-10774 & 1720 & 70 & $0.0 \pm 2.0 \mathrm{E}$ & $1150-1390$ & Marine shell & Tridacna maxima & 15: $20-30 \mathrm{cmbs}$ & ANU-EPF \\
\hline Ugaga & Ugaga & ANU-10775 & 2620 & 60 & $0.0 \pm 2.0 \mathrm{E}$ & $2130-2470$ & Marine shell & Tridacna maxima & 19: $30-40 \mathrm{cmbs}$ & ANU-EPF \\
\hline Ugaga & Ugaga & ANU-10776 & 1900 & 60 & $0.0 \pm 2.0 \mathrm{E}$ & $1310-1590$ & Marine shell & Turbo argyrostromus & M8: $20-30 \mathrm{cmbs}$ & ANU-EPF \\
\hline Ugaga & Ugaga & ANU-10777 & 2530 & 70 & $0.0 \pm 2.0 \mathrm{E}$ & $2000-2340$ & Marine shell & Tridacna maxima & J9: $40-50 \mathrm{cmbs}$ & ANU-EPF \\
\hline Ugaga & Ugaga & ANU-10778 & 2600 & 70 & $0.0 \pm 2.0 \mathrm{E}$ & $2090-2470$ & Marine shell & Tridacna maxima & $\mathrm{T} / \mathrm{U}-1: 40-50 \mathrm{cmbs}$ & ANU-EPF \\
\hline Ugaga & Ugaga & Beta-107951 & 2130 & 50 & 2.0 & $1580-1850$ & Marine shell & Tridacna maxima & minus $A 13: 30-40 \mathrm{cmbs}$ & ANU-EPF \\
\hline Ugaga & Ugaga & Beta-107952 & 2690 & 60 & 2.0 & $2260-2650$ & Marine shell & Tridacna maxima & P10: $30-40 \mathrm{cmbs}$ & ANU-EPF \\
\hline Ugaga & Ugaga & Beta-107953 & 3150 & 70 & 3.2 & $2760-3140$ & Marine shell & Trochus niloticus & $\mathrm{C} 12: 50-60 \mathrm{cmbs}$ & ANU-EPF \\
\hline Ugaga & Ugaga & Wk-5554 & $98.3 \pm 0.5 \% \mathrm{M}$ & - & $-24 \pm 2.0 \mathrm{E}$ & - & Charcoal & - & Sq. K7: 20-30 & ANU-EPF \\
\hline Ugaga & Ugaga & Wk-5555 & $98.8 \pm 0.5 \% \mathrm{M}$ & - & $-24 \pm 2.0 \mathrm{E}$ & - & Charcoal & - & Sq. K7: $20-30$ & ANU-EPF \\
\hline Ugaga & Ugaga & Wk-5556 & $98.2 \pm 0.5 \% \mathrm{M}$ & - & $-24 \pm 2.0 \mathrm{E}$ & - & Charcoal & - & Sq. P8: $20-30$ & ANU-EPF \\
\hline Vanuabalavu & Qaranilaca & ANU-11536H & 980 & 80 & $0.0 \pm 2.0$ & $460-690$ & Marine shell & Anadara sp. & Layer III: 80 cmbs & Nunn et al. (2004a) \\
\hline Vanuabalavu & Qaranilaca & ANU-11537H & 1140 & 80 & $0.0 \pm 2.0$ & $550-870$ & Marine shell & Anadara sp. & Layer IV: 105 cmbs & Nunn et al. (2004a) \\
\hline Vanuabalavu & Qaranilaca & ANU-11538H & 1060 & 60 & $-24 \pm 2.0 \mathrm{E}$ & $790-1060$ & Charcoal & - & Layer III: $75-85 \mathrm{cmbs}$ & Nunn et al. (2004a) \\
\hline Vanuabalavu & Qaranilaca & ANU-11539H & 1180 & 60 & $-24 \pm 2.0 \mathrm{E}$ & $930-1220$ & Charcoal & - & Layer IV: $104-109 \mathrm{cmbs}$ & Nunn et al. (2004a) \\
\hline Viti Levu & Bourewa & Wk-14235 & 2896 & 40 & -27.4 & $2810-3140$ & Charcoal & - & Pit 3: $123 \mathrm{cmbs}$ & P. Nunn, pers. comm., USP \\
\hline Viti Levu & Bourewa & Wk-14236 & 2867 & 40 & -25.0 & $2790-3060$ & Charcoal & - & Pit 3: $109 \mathrm{cmbs}$ & P. Nunn, pers. comm., USP \\
\hline Viti Levu & Bourewa & Wk-14237 & 3259 & 40 & -12.8 & $2950-3220$ & Marine shell & Tellinidae & Pit 4: $53 \mathrm{cmbs}$ & P. Nunn, pers. comm., USP \\
\hline Viti Levu & Bourewa & Wk-14238 & 2740 & 40 & $2.5 \pm 0.2$ & $2330-2640$ & Marine shell & $\begin{array}{l}\text { Fimbria fimbriata, } \\
\text { Codakia punctata }\end{array}$ & Pit 2: $55 \mathrm{cmbs}$ & P. Nunn, pers. comm., USP \\
\hline Viti Levu & Bourewa & Wk-14239 & 3027 & 40 & $3.5 \pm 0.2$ & $2720-2900$ & Marine shell & Trochus niloticus & Pit 1: $71 \mathrm{cmbs}$ & P. Nunn, pers. comm., USP \\
\hline Viti Levu & Bourewa & Wk-14594 & 2944 & 40 & $2.9 \pm 0.2$ & $2610-2830$ & Marine shell & Codakia punctata & Pit 3: $105 \mathrm{cmbs}$ & P. Nunn, pers. comm., USP \\
\hline Viti Levu & Bourewa & Wk-14595 & 2915 & 40 & -23.5 & $2860-3140$ & Charcoal & - & Pit 4: $54 \mathrm{cmbs}$ & P. Nunn, pers. comm., USP \\
\hline Viti Levu & Bourewa & Wk-14597 & 2717 & 40 & -23.6 & $2730-2860$ & Charcoal & - & Pit 2: $57 \mathrm{cmbs}$ & P. Nunn, pers. comm., USP \\
\hline Viti Levu & Bourewa & Wk-14598 & 2612 & 40 & -23.1 & $2490-2760$ & Charcoal & - & Pit 1: $54 \mathrm{cmbs}$ & P. Nunn, pers. comm., USP \\
\hline Viti Levu & Bourewa & Wk-14599 & 2894 & 40 & -24.7 & $2800-3140$ & Charcoal & - & Pit 1: $85 \mathrm{cmbs}$ & P. Nunn, pers. comm., USP \\
\hline Viti Levu & Bourewa & Wk-16206 & 1590 & 40 & $3.6 \pm 0.2$ & $1050-1250$ & Marine shell & $\begin{array}{l}\text { Strombus gibberulus, } \\
\text { Strombus labiatus, Fimbria } \\
\text { fimbriata, } \\
\text { Vasum turbinellus }\end{array}$ & Pit L4: 82 cmbs & P. Nunn, pers. comm., USP \\
\hline Viti Levu & Bourewa & Wk-16207 & 2940 & 40 & $2.9 \pm 0.2$ & $2610-2730$ & Marine shell & Anadara sp., Codakia sp. & Pit B3: $90 \mathrm{cmbs}$ & P. Nunn, pers. comm., USP \\
\hline Viti Levu & Bourewa & Wk-16216 & 164 & 40 & -24.4 & $0-280$ & Charcoal & - & Pit B4: $134 \mathrm{cmbs}$ & P. Nunn, pers. comm., USP \\
\hline Viti Levu & Bourewa & Wk-17539 & 383 & 30 & -25.1 & $320-490$ & Charcoal & - & Pit X8: $95 \mathrm{cmbs}$ & P. Nunn, pers. comm., USP \\
\hline Viti Levu & Bourewa & Wk-17540 & 133 & 30 & -26.7 & $0-260$ & Charcoal & - & Pit X2: $196 \mathrm{cmbs}$ & P. Nunn, pers. comm., USP \\
\hline Viti Levu & Bourewa & Wk-17541 & 2506 & 30 & -26.1 & $2360-2710$ & Charcoal & - & Pit X1: $105 \mathrm{cmbs}$ & P. Nunn, pers. comm., USP \\
\hline Viti Levu & Bourewa & Wk-17542 & 2920 & 30 & -24.4 & $2870-3140$ & Charcoal & - & Pit X3: $135 \mathrm{cmbs}$ & P. Nunn, pers. comm., USP \\
\hline
\end{tabular}


Table 19 continued

\begin{tabular}{|c|c|c|c|c|c|c|c|c|c|c|}
\hline Island & Site & Lab Code & CRA & CRA SE & C13 & Cal. BP & $\begin{array}{l}\text { Sample } \\
\text { Type }\end{array}$ & Identification & Context & Reference \\
\hline Viti Levu & Bourewa & Wk-17543 & 3006 & 40 & $4.1 \pm 0.2$ & $2710-2870$ & Marine shell & Trochus niloticus & Pit X24E: $52 \mathrm{cmbs}$ & P. Nunn, pers. comm., USP \\
\hline Viti Levu & Bourewa & Wk-17544 & 3474 & 40 & $3.2 \pm 0.2$ & $3250-3450$ & Marine shell & Conus sp. & Pit X25: $140 \mathrm{cmbs}$ & P. Nunn, pers. comm., USP \\
\hline Viti Levu & Bourewa & Wk-17545 & 2851 & 40 & $2.9 \pm 0.2$ & $2470-2720$ & Marine shell & Codakia punctata & Pit X23: $98 \mathrm{cmbs}$ & P. Nunn, pers. comm., USP \\
\hline Viti Levu & Bourewa & Wk-17546 & 2951 & 40 & $2.4 \pm 0.2$ & $2650-2840$ & Marine shell & $\begin{array}{l}\text { Codakia punctata, } \\
\text { Gafrarium tumidum, } \\
\text { Turbo sp. }\end{array}$ & Pit X3: $124 \mathrm{cmbs}$ & P. Nunn, pers. comm., USP \\
\hline Viti Levu & Bourewa & Wk-17547 & 3006 & 40 & $4.5 \pm 0.2$ & $2710-2870$ & Marine shell & Trochus niloticus & Pit X6: $94 \mathrm{cmbs}$ & P. Nunn, pers. comm., USP \\
\hline Viti Levu & Bourewa & Wk-17548 & 2938 & 40 & $1.6 \pm 0.2$ & $2610-2820$ & Marine shell & Anadarasp. & Pit X2: $120 \mathrm{cmbs}$ & P. Nunn, pers. comm., USP \\
\hline Viti Levu & Bourewa & Wk-17549 & 3046 & 40 & $4.4 \pm 0.2$ & $2730-2920$ & Marine shell & Trochus niloticus & Pit X3: 104 cmbs & P. Nunn, pers. comm., USP \\
\hline Viti Levu & Bourewa & Wk-17967 & 198 & 30 & -19.4 & $0-130$ & Bone & Sus scrofa & Pit X8: $55 \mathrm{cmbs}$ & P. Nunn, pers. comm., USP \\
\hline Viti Levu & Bourewa & Wk-17968 & 3107 & 40 & $2.4 \pm 0.2$ & $2760-3000$ & Marine shell & Atactodea striata & Pit X6: $68 \mathrm{cmbs}$ & P. Nunn, pers. comm., USP \\
\hline Viti Levu & Bourewa & Wk-17969 & 1740 & 30 & $2.9 \pm 0.2$ & $1230-1360$ & Marine shell & Strombus gibberulus & Pit X8: $68 \mathrm{cmbs}$ & P. Nunn, pers. comm., USP \\
\hline Viti Levu & Bourewa & Wk-17970 & 2866 & 30 & $2.3 \pm 0.2$ & $2610-2730$ & Marine shell & Tridacna squamosa & Pit X10: $78 \mathrm{cmbs}$ & P. Nunn, pers. comm., USP \\
\hline Viti Levu & Bourewa & Wk-17971 & 2831 & 40 & $3.8 \pm 0.2$ & $2450-2710$ & Marine shell & Turbo chrysostomus & Pit X20: $60 \mathrm{cmbs}$ & P. Nunn, pers. comm., USP \\
\hline Viti Levu & Bourewa & Wk-17972 & 2824 & 40 & $3.0 \pm 0.2$ & $2430-2710$ & Marine shell & Turbo sp. & Pit X20: $69 \mathrm{cmbs}$ & P. Nunn, pers. comm., USP \\
\hline Viti Levu & Bourewa & Wk-17973 & 2870 & 30 & -25.8 & $2800-3060$ & Charcoal & - & Pit X3: $121 \mathrm{cmbs}$ & P. Nunn, pers. comm., USP \\
\hline Viti Levu & Bourewa & Wk-17974 & 891 & 30 & -25.2 & $680-900$ & Charcoal & - & Pit X21E: $45 \mathrm{cmbs}$ & P. Nunn, pers. comm., USP \\
\hline Viti Levu & Bourewa & Wk-20281 & 3038 & 40 & $3.6 \pm 0.2$ & $2730-2910$ & Marine shell & Trochus niloticus & Pit X2: $90 \mathrm{cmbs}$ & P. Nunn, pers. comm., USP \\
\hline Viti Levu & Bourewa & Wk-20282 & 3014 & 40 & $3.6 \pm 0.2$ & $2710-2880$ & Marine shell & Trochus niloticus & Pit X2: $20 \mathrm{cmbs}$ & P. Nunn, pers. comm., USP \\
\hline Viti Levu & Bourewa & Wk-20283 & 2976 & 40 & $4.3 \pm 0.2$ & $2690-2850$ & Marine shell & Trochus niloticus & Pit X3: $70 \mathrm{cmbs}$ & P. Nunn, pers. comm., USP \\
\hline Viti Levu & Bourewa & Wk-20284 & 3044 & 40 & $3.6 \pm 0.2$ & $2730-2920$ & Marine shell & Trochus niloticus & Pit X3: $30 \mathrm{cmbs}$ & P. Nunn, pers. comm., USP \\
\hline Viti Levu & Bukusia & AA-50303 & 189 & 50 & -28.3 & $0-300$ & Charcoal & - & TU $1,30-40 \mathrm{cmbs}$ & Field (2004: 90) \\
\hline Viti Levu & Bukusia & AA-50304 & 259 & 40 & -24.3 & $0-440$ & Charcoal & - & TU $2,40-50 \mathrm{cmbs}$ & Field (2004: 90) \\
\hline Viti Levu & Bukusia & Wk-11135 & 202 & 60 & -29.6 & $0-300$ & Charcoal & - & TU 2, $80-90 \mathrm{cmbs}$ & Field (2004: 90) \\
\hline Viti Levu & Korohewa & AA-50305 & 210 & 40 & -27.9 & $0-300$ & Charcoal & - & TU $1,60-70 \mathrm{cmbs}$ & Field (2004: 90) \\
\hline Viti Levu & Korovatuma & AA-50300 & 527 & 50 & -27.2 & $460-630$ & Charcoal & - & TU $1,60-70 \mathrm{cmbs}$ & Field (2004: 90) \\
\hline Viti Levu & Korovatuma & AA-50301 & 188 & 40 & -27.2 & $0-290$ & Charcoal & - & TU $1,20-30 \mathrm{cmbs}$ & Field (2004: 90) \\
\hline Viti Levu & Korovatuma & AA-50310 & 338 & 50 & -26.0 & $160-490$ & Charcoal & - & TU 2, $60 \mathrm{cmbs}$ & Field (2004: 90) \\
\hline Viti Levu & Madraya & AA-50296 & 398 & 40 & -28.7 & $320-500$ & Charcoal & - & TU1, 20-30 cmbs & Field (2004: 90) \\
\hline Viti Levu & Malaqerqere & ANU-10452 & 460 & 60 & $-24 \pm 2.0 \mathrm{E}$ & $320-540$ & Charcoal & - & Sq. A1: $10-20 \mathrm{cmbs}$ & ANU-EPF \\
\hline Viti Levu & Malaqerqere & ANU-10453 & 830 & 90 & $-24 \pm 2.0 \mathrm{E}$ & $560-910$ & Charcoal & - & Sq. $A 2: 60-70 \mathrm{cmbs}$ & ANU-EPF \\
\hline Viti Levu & Malaqerqere & ANU-10454 & 670 & 70 & $-24 \pm 2.0 \mathrm{E}$ & $520-680$ & Charcoal & - & Sq. $A 1: 40-50 \mathrm{cmbs}$ & ANU-EPF \\
\hline Viti Levu & Malua & AA-50290 & 159 & 30 & -26.5 & $0-280$ & Charcoal & - & TU1, 50-60 cmbs & Field (2004: 90) \\
\hline Viti Levu & Malua & AA-50295 & 624 & 30 & -26.3 & $530-640$ & Charcoal & - & TU2, $10-20 \mathrm{cmbs}$ & Field (2004: 90) \\
\hline Viti Levu & Nadroga & AA-50297 & 224 & 50 & -24.6 & $0-320$ & Charcoal & - & TU1, 50-60 cmbs & Field (2004: 90) \\
\hline Viti Levu & Nasilai & Beta-22096 & Modern & - & - & - & Wood & Post & Level IV & Rosenthal (1995: 97) \\
\hline Viti Levu & Nasilai & Beta-22097 & 330 & 70 & - & $150-230$ & Wood & Canoe mast & $?$ & Rosenthal (1995: 97) \\
\hline Viti Levu & Natunuku & ANU-10698 & 2780 & 90 & $0.0 \pm 2.0 \mathrm{E}$ & $2310-2720$ & Marine shell & Tridacna sp. & Tr.3, Sq.A5: $30-40 \mathrm{cmbs}$ & ANU-EPF \\
\hline
\end{tabular}


Table 19 continued

\begin{tabular}{|c|c|c|c|c|c|c|c|c|c|c|}
\hline Island & Site & Lab Code & CRA & CRA SE & $\mathrm{C} 13$ & Cal. BP & $\begin{array}{l}\text { Sample } \\
\text { Type }\end{array}$ & Identification & Context & Reference \\
\hline Viti Levu & Natunuku & ANU-10699 & 1160 & 70 & $0.0 \pm 2.0 \mathrm{E}$ & $600-890$ & Marine shell & Tridacna sp. & Tr.3, Sq.A5: $30-40 \mathrm{cmbs}$ & ANU-EPF \\
\hline Viti Levu & Natunuku & ANU-10700 & 380 & 70 & $0.0 \pm 2.0 \mathrm{E}$ & $0-230$ & Marine shell & Trochus sp. & Tr.3, Sq.A5: $20-30 \mathrm{cmbs}$ & ANU-EPF \\
\hline Viti Levu & Natunuku & ANU-11305 & 2900 & 50 & $0.0 \pm 2.0 \mathrm{E}$ & $2490-2770$ & Marine shell & ?Species & Trench 3 & ANU-EPF \\
\hline Viti Levu & Natunuku & ANU-11306 & 1170 & 50 & $0.0 \pm 2.0 \mathrm{E}$ & $630-840$ & Marine shell & ?Species & Trench 3 & ANU-EPF \\
\hline Viti Levu & Natunuku & ANU-11307 & 2600 & 60 & $0.0 \pm 2.0 \mathrm{E}$ & $2110-2440$ & Marine shell & ? Species & Trench 3 & ANU-EPF \\
\hline Viti Levu & Natunuku & GaK-1218 & 3240 & 100 & - & $3080-3680$ & Charcoal & - & $\begin{array}{l}\text { Layer 6, Rectangle E, } \\
\text { Location C }\end{array}$ & Davidson et al. (1990: 131) \\
\hline Viti Levu & Natunuku & NZ-7863 & 2640 & 30 & 0.7 & $2210-2440$ & Marine shell & Tridacna maxima & $\begin{array}{l}\text { Layer 5, Rectangle D, } \\
\text { Location C }\end{array}$ & Petchey (1995: 94) \\
\hline Viti Levu & Natunuku & NZ-7864 & 2750 & 30 & 0.3 & $2340-2610$ & Marine shell & Tridacna maxima & $\begin{array}{l}\text { Layer 5b, Rectangle D, } \\
\text { Location C }\end{array}$ & Petchey (1995: 94) \\
\hline Viti Levu & Natunuku & NZ-7865 & 2622 & 30 & -0.3 & $2180-2390$ & Marine shell & Gafarium tumidum & $\begin{array}{l}\text { Layer } 5 b \text {, Rectangle D, } \\
\text { Location C }\end{array}$ & Petchey (1995: 94) \\
\hline Viti Levu & Natunuku & NZA-2117 & 2676 & 60 & 1.8 & $2200-2600$ & Marine shell & Gafarium tumidum & $\begin{array}{l}\text { Layer 5, Rectangle D, } \\
\text { Location C }\end{array}$ & Petchey (1995: 94) \\
\hline Viti Levu & Natunuku & NZA-2512 & 1896 & 90 & -14.8 & $1400-1820$ & Bone & Human & $\begin{array}{l}\text { Rectangle C, Layer above 5, } \\
\text { Location C }\end{array}$ & Davidson et al. (1990: 131) \\
\hline Viti Levu & Navatu & M-5810 & 950 & 300 & - & $320-1390$ & Charcoal & - & $24-30$ inches bs & Gifford (1951b) \\
\hline Viti Levu & Navatu & M-5879 & 1200 & 500 & - & $150-2150$ & Charcoal & - & 90 inches bs & Gifford (1951b) \\
\hline Viti Levu & Navatu & M-6342 & 1300 & 500 & - & $310-2310$ & Charcoal & - & 96-104 inches bs & Gifford (1951b) \\
\hline Viti Levu & Navatu & M-351 & 2000 & 500 & $-24 \pm 2.0 \mathrm{E}$ & $850-3140$ & Charcoal & - & $104-110$ inches bs & Gifford (1951b) \\
\hline Viti Levu & Navatu 17A & ANU-10384 & 870 & 70 & $-24 \pm 2.0 \mathrm{E}$ & $660-910$ & Charcoal & - & Tr. B1: L2, $140 \mathrm{cmbs}$ & ANU-EPF \\
\hline Viti Levu & Navatu 17A & ANU-10385 & 350 & 70 & $-24 \pm 2.0 \mathrm{E}$ & $150-500$ & Charcoal & - & Tr. A1: L1, $100 \mathrm{cmbs}$ & ANU-EPF \\
\hline Viti Levu & Navatu 17A & ANU-10386 & 1670 & 70 & $-24 \pm 2.0 \mathrm{E}$ & $1350-1690$ & Charcoal & - & Tr. B3+4: L4, $230 \mathrm{cmbs}$ & ANU-EPF \\
\hline Viti Levu & Navatu 17A & ANU-10387 & 1240 & 140 & $-24 \pm 2.0 \mathrm{E}$ & $800-1350$ & Charcoal & - & Tr. B1+2: L4, $215 \mathrm{cmbs}$ & ANU-EPF \\
\hline Viti Levu & Navatu 17A & ANU-10389 & 330 & 60 & $-24 \pm 2.0 \mathrm{E}$ & $150-490$ & Charcoal & - & Tr. B1: L1, 86 cmbs & ANU-EPF \\
\hline Viti Levu & Navatu 17A & ANU-10390 & 1010 & 140 & $-24 \pm 2.0 \mathrm{E}$ & $660-1180$ & Charcoal & - & Tr. B1+2: L4, $220 \mathrm{cmbs}$ & ANU-EPF \\
\hline Viti Levu & Navatu 17A & ANU-10708 & 1980 & 70 & $0.0 \pm 2.0 \mathrm{E}$ & $1360-1710$ & Marine shell & Tridacna sp. & Tr. B3+4: L4, 230 cmbs & ANU-EPF \\
\hline Viti Levu & Navatu 17A & ANU-10709 & 980 & 70 & $0.0 \pm 2.0 \mathrm{E}$ & $480-670$ & Marine shell & Gafrarium sp. & Tr. B1: L2, $120 \mathrm{cmbs}$ & ANU-EPF \\
\hline Viti Levu & Navatu 17A & ANU-10710 & 720 & 70 & $0.0 \pm 2.0 \mathrm{E}$ & $250-500$ & Marine shell & Anadara sp. & Tr. B2: L1, $50 \mathrm{cmbs}$ & ANU-EPF \\
\hline Viti Levu & Nokonoko & AA-50283 & 1311 & 40 & -25.0 & $1080-1280$ & Charcoal & - & TU 1, $95-105 \mathrm{cmbs}$ & Field (2004: 90) \\
\hline Viti Levu & Nokonoko & AA-50284 & 674 & 40 & -8.7 & $440-550$ & Riparian shell & ?Species & TU $3,10-20 \mathrm{cmbs}$ & Field (2004: 90) \\
\hline Viti Levu & Nokonoko & AA-50287 & 1492 & 40 & -26.7 & $1290-1400$ & Charcoal & - & TU 5, 20-30 cmbs & Field (2004: 90) \\
\hline Viti Levu & Nokonoko & AA-50288 & 212 & 40 & -24.5 & $0-310$ & Charcoal & - & TU 6, 30-40 cmbs & Field (2004: 90) \\
\hline Viti Levu & Nokonoko & AA-50289 & 135 & 30 & -24.9 & $0-260$ & Charcoal & - & TU $10,10-20 \mathrm{cmbs}$ & Field (2004: 90) \\
\hline Viti Levu & Nokonoko & AA-50299 & 185 & 60 & -27.4 & $0-290$ & Charcoal & - & TU 7, $30-40 \mathrm{cmbs}$ & Field (2004: 90) \\
\hline Viti levu & Qara-I-Oso I & ANU-11015 & 1280 & 120 & $-24 \pm 2.0 \mathrm{E}$ & $920-1350$ & Charcoal & - & TP. $1: 30 \mathrm{cmbs}$ & ANU-EPF \\
\hline Viti Levu & Qara-I-Oso II & ANU-11014 & 660 & 60 & $-24 \pm 2.0 \mathrm{E}$ & $530-670$ & Charcoal & - & TP.1, Layer 2: $30 \mathrm{cmbs}$ & ANU-EPF \\
\hline Viti Levu & Qaramatatolu & Wk-16214 & 297 & 40 & - & $150-450$ & Charcoal & - & Pit M4: $160 \mathrm{cmbs}$ & Nunn et al. (2005) \\
\hline Viti Levu & Qaramatatolu & Wk-16213 & 970 & 40 & - & $750-920$ & Charcoal & - & Pit M4: $145 \mathrm{cmbs}$ & Nunn et al. (2005) \\
\hline
\end{tabular}


Table 19 continued

\begin{tabular}{|c|c|c|c|c|c|c|c|c|c|c|}
\hline Island & Site & Lab Code & CRA & CRA SE & C13 & Cal. BP & $\begin{array}{l}\text { Sample } \\
\text { Type }\end{array}$ & Identification & Context & Reference \\
\hline Viti Levu & Qoroqoro & AA-50307 & 974 & 40 & -29.4 & $750-930$ & Charcoal & - & TU1, $90-100 \mathrm{cmbs}$ & Field (2004: 90) \\
\hline Viti Levu & Qoroqoro & AA-50308 & 182 & 40 & -29.1 & $0-290$ & Charcoal & - & TU3, 20-30 cmbs & Field (2004: 90) \\
\hline Viti Levu & Qoroqoro & Wk-11134 & 259 & 100 & -28.2 & $0-460$ & Charcoal & - & TU4, 50-70 cmbs & Field (2004) \\
\hline Viti Levu & Rove & Wk-14240 & 155 & 30 & -25.2 & $0-280$ & Charcoal & - & Pit 1: $65 \mathrm{cmbs}$ & P. Nunn, pers. comm., USP \\
\hline Viti Levu & Rove & Wk-14241 & 187 & 60 & -24.9 & $0-290$ & Charcoal & - & Pit 1: $95 \mathrm{cmbs}$ & P. Nunn, pers. comm., USP \\
\hline Viti Levu & Rove & Wk-14242 & 95 & 40 & -24.6 & - & Charcoal & - & Pit $1: 75 \mathrm{cmbs}$ & P. Nunn, pers. comm., USP \\
\hline Viti Levu & Rukuruku & Beta 64460 & 210 & 60 & - & - & Marine shell & Trochus niloticus & Surface & Kuhlken and Crosby (1999) \\
\hline Viti Levu & Rukuruku & Beta 64461 & 160 & 70 & - & $0-290$ & Charcoal & - & Trench 3/4, Layer 3: $60 \mathrm{cmbs}$ & Kuhlken and Crosby (1999) \\
\hline Viti Levu & Sigatoka & CAMS 32251 & 230 & 40 & -27.8 & $0-310$ & Charcoal & - & West Dunes & Dickinson et al. (1998) \\
\hline Viti Levu & Sigatoka & CAMS 32252 & 510 & 60 & -25.9 & $330-630$ & Charcoal & - & Open field & Dickinson et al. (1998) \\
\hline Viti Levu & Sigatoka & CAMS 48565 & 2470 & 50 & -23.7 & $2350-2700$ & Charcoal & - & Level 1 & Burley $(2003,2005)$ \\
\hline Viti Levu & Sigatoka & CAMS 48566 & 2490 & 50 & -21.4 & $2350-2710$ & Charcoal & - & Level 1 & Burley $(2003,2005)$ \\
\hline Viti Levu & Sigatoka & CAMS 68191 & 1550 & 40 & -27.4 & $1300-1520$ & Charcoal & - & Level 2 & Burley $(2003,2005)$ \\
\hline Viti Levu & Sigatoka & CAMS 68192 & 1540 & 40 & -26 & $1300-1520$ & Charcoal & - & Level 2 & Burley $(2003,2005)$ \\
\hline Viti Levu & Sigatoka & CAMS 68194 & 1620 & 40 & -25.5 & $1360-1540$ & Charcoal & - & Level 2 & Burley $(2003,2005)$ \\
\hline Viti Levu & Sigatoka & CAMS 68195 & 1310 & 40 & 24.5 & $1080-1280$ & Charcoal & - & Level 2 & Burley $(2003,2005)$ \\
\hline Viti Levu & Sigatoka & CAMS 68196 & 2510 & 40 & -24.3 & $2360-2710$ & Charcoal & - & Level 1 & Burley $(2003,2005)$ \\
\hline Viti Levu & Sigatoka & CAMS 70090 & 1400 & 40 & -28.3 & $1180-1330$ & Charcoal & - & Level 2 & Burley $(2003,2005)$ \\
\hline Viti Levu & Sigatoka & CAMS 70091 & 1430 & 40 & -27.4 & $1180-1360$ & Charcoal & - & Level 2 & Burley $(2003,2005)$ \\
\hline Viti Levu & Sigatoka & CAMS 70920 & 1480 & 40 & -25.5 & $1280-1390$ & Charcoal & - & Level 2 & Burley $(2003,2005)$ \\
\hline Viti Levu & Sigatoka & CAMS 70921 & 1410 & 40 & -24.6 & $1180-1340$ & Charcoal & - & Level 2 & Burley $(2003,2005)$ \\
\hline Viti Levu & Sigatoka & GaK-1206 & 1720 & 80 & - & 1380-1810 & Charcoal & - & $\begin{array}{l}\text { Level 2, Sq. 52C, 12-15 } \\
\text { inches }\end{array}$ & Birks (1973:57) \\
\hline Viti Levu & Sigatoka & GaK-946 & 2460 & 90 & - & $2190-2740$ & Charcoal & - & $\begin{array}{l}\text { Level } 1 \text {, Sq. } 45 \mathrm{~A} \text {, bottom } \\
6 \text { inches }\end{array}$ & Birks (1973:57) \\
\hline Viti Levu & Sigatoka & NZ-7599 & 1680 & 60 & - & $1380-1690$ & Charcoal & - & Level 2 & Best (1988) \\
\hline Viti Levu & Sigatoka & NZA-4789 & 2627 & 80 & -25.8 & $2360-2840$ & Charcoal & - & Level 1',53 cm below datum & Petchey (1995) \\
\hline Viti Levu & Sigatoka & Wk 8239 & 2740 & 80 & - & $2500-3000$ & Charcoal & - & Level 1 & De Biran (2001) \\
\hline Viti Levu & Sigatoka & Wk 8328 & 1410 & 150 & - & $960-1550$ & Charcoal & - & Level 2 & De Biran (2001) \\
\hline Viti Levu & Sigatoka & Wk 8900 & 690 & 50 & - & $550-670$ & Charcoal & - & Level 3 & De Biran (2001) \\
\hline Viti Levu & Sigatoka & Wk 9030 & 1400 & 40 & - & $1180-1330$ & Charcoal & - & Level 2 & De Biran (2001) \\
\hline Viti Levu & Sigatoka & WK 996a & Modern & - & -7.6 & - & Bone & Human & Mound south of Sq. B4 & Best $(1988: 6,14)$ \\
\hline Viti Levu & Sigatoka & WK 996b & 1870 & 70 & -15.9 & 1390-1770 & Bone & Human & Mound south of Sq. B4 & Best $(1988: 6,14)$ \\
\hline Viti Levu & Sigatoka & Wk-5333 & 590 & 60 & -26.3 & $500-650$ & Charcoal & - & West Dune Field: Level 3? & Dickinson et al. (1998) \\
\hline Viti Levu & Tatuba Cave & AA-50291 & 207 & 30 & -28.1 & $0-300$ & Charcoal & - & TU1, 190-200 cmbs & Field (2004: 90) \\
\hline Viti Levu & Tatuba Cave & AA-50292 & 1802 & 30 & -25.5 & $1550-1770$ & Charcoal & - & TU1, $140-150 \mathrm{cmbs}$ & Field (2004: 90) \\
\hline Viti Levu & Tatuba Cave & AA-50293 & 339 & 40 & -20.6 & $0-260$ & Bone & Human & TU $1,130-140 \mathrm{cmbs}$ & Field (2004: 90) \\
\hline Viti Levu & Tatuba Cave & AA-50294 & 1993 & 40 & -26.0 & 1740-1990 & Charcoal & - & TU1, 80-90 cmbs & Field (2004: 90) \\
\hline Viti Levu & Tatuba Cave & AA-50298 & 1294 & 40 & -25.6 & $1070-1270$ & Charcoal & - & TU1, 50-60 cmbsr & Field (2004: 90) \\
\hline
\end{tabular}


Table 19 continued

\begin{tabular}{|c|c|c|c|c|c|c|c|c|c|c|}
\hline Island & Site & Lab Code & CRA & CRA SE & C13 & Cal. BP & $\begin{array}{l}\text { Sample } \\
\text { Type }\end{array}$ & Identification & Context & Reference \\
\hline Viti Levu & Tatuba Cave & Wk-11137 & 968 & 50 & -28.0 & $740-930$ & Charcoal & - & TU1, Ft. $1,45-55$ cmbs & Field (2004: 90) \\
\hline Viti Levu & Tomato patch & Wk-16205 & 1720 & 30 & $3.5 \pm 0.2$ & $1210-1340$ & Marine shell & Trochus sp. & Surface: 88 cmbs & P. Nunn, pers. comm., USP \\
\hline Viti Levu & Tomato patch & Wk-16215 & 370 & 40 & -26.9 & $310-490$ & Charcoal & - & Surface: 90 cmbs & P. Nunn, pers. comm., USP \\
\hline Viti Levu & Tuvu & ANU-11020 & 1570 & 100 & -24.0 & $1260-1700$ & Charcoal & - & TP 1, Spit IV & ANU-EPF \\
\hline Viti Levu & Vitoga & AA-50302 & 198 & 60 & -24.9 & $0-300$ & Charcoal & - & TU1, 60-77 cmbs & Field (2004: 90) \\
\hline Viti Levu & Vitoga & AA-50306 & 175 & 40 & -25.0 & $0-280$ & Charcoal & - & TU1, 20-30 cmbs & Field (2004: 90) \\
\hline Viti Levu & Vitoga & AA-50309 & 261 & 50 & -25.1 & $0-450$ & Charcoal & - & TU2, 40-50 cmbs & Field (2004: 90) \\
\hline Viti Levu & Volivoli Il & ANU-10449 & 1120 & 70 & -24.0 & 810-1170 & Charcoal & - & TR. 1: $20-30 \mathrm{cmbs}$ & ANU-EPF \\
\hline Viti Levu & Volivoli Il & ANU-10450 & 1960 & 70 & -24.0 & $1630-2040$ & Charcoal & - & TR. 1: $40-50 \mathrm{cmbs}$ & ANU-EPF \\
\hline Viti Levu & Volivoli Il & ANU-10451 & 1080 & 190 & -24.0 & 580-1300 & Charcoal & - & TR.1: 50-60 cmbs & ANU-EPF \\
\hline Viti Levu & Volivoli III & ANU-11016 & 1100 & 90 & -24.0 & 770-1170 & Charcoal & - & TP. 1, Spit 5 & ANU-EPF \\
\hline Viti Levu & Volivoli III & ANU-11018 & 290 & 60 & -24.0 & $0-490$ & Charcoal & - & TP. 1, Spit 2 & ANU-EPF \\
\hline Viti Levu & Volivoli III & ANU-11019 & 1060 & 80 & -24.0 & $740-1070$ & Charcoal & - & TP. 1, Spit 8 & ANU-EPF \\
\hline Viti Levu & Vunda & $? 6349$ & 700 & 300 & - & $0-1170$ & Charcoal & - & 24-30 inches bs & Gifford (1951b) \\
\hline Viti Levu & Vunda & $? 6353$ & 650 & 300 & - & $0-1060$ & Charcoal & - & 12-18 inches bs & Gifford (1951b) \\
\hline Viti Levu & Yanuca & ANU-11413 & 2650 & 50 & $-12.3 \pm 0.2$ & $2170-2490$ & Marine shell & $\begin{array}{l}\text { Trachycardium cf. } \\
\text { reeveanum }\end{array}$ & $\begin{array}{l}\text { Trench 4, Zone 3, Spit 2, } \\
152.4 \text { cmbs }\end{array}$ & Clark and Anderson (2001) \\
\hline Viti Levu & Yanuca & ANU-11414 & 3150 & 60 & $-12.3 \pm 2.0$ & $2780-3120$ & $\begin{array}{l}\text { Fresh water } \\
\text { shell }\end{array}$ & Batissa violacea & $\begin{array}{l}\text { Trench 2A, Zone 3, Spit 4, } \\
167.6 \mathrm{cmbs}\end{array}$ & Clark and Anderson (2001) \\
\hline Viti Levu & Yanuca & ANU-11415 & 2300 & 50 & $1.5 \pm 2.0$ & $1790-2060$ & Marine shell & Tonna sulcosa & $\begin{array}{l}\text { Trench 3A, Zone 3, Spit 4, } \\
167.6 \mathrm{cmbs}\end{array}$ & Clark and Anderson (2001) \\
\hline Viti Levu & Yanuca & ANU-11416 & 2940 & 60 & $3.8 \pm 2.0$ & $2520-2850$ & Marine shell & Anadara antiquata & $\begin{array}{l}\text { Trench 3, Zone 3, Spit 7, } \\
190.5 \text { cmbs }\end{array}$ & Clark and Anderson (2001) \\
\hline Viti Levu & Yanuca & ANU-11417 & 3050 & 80 & $2.7 \pm 5.7$ & $2680-3050$ & Marine shell & Cyprea tigris & $\begin{array}{l}\text { Trench 2-2A, Zone 3, Spit 3, } \\
160.0 \mathrm{cmbs}\end{array}$ & Clark and Anderson (2001) \\
\hline Viti Levu & Yanuca & GaK-1226 & 2980 & 90 & - & $2860-3340$ & Charcoal & - & Zone 3,66 inches bs & Birks and Birks (1978) \\
\hline Viti Levu & Yanuca & GaK-1227 & 2660 & 90 & - & $2360-2880$ & Charcoal & - & Zone 2,48 inches bs & Birks and Birks (1978) \\
\hline Viti Levu & Yanuca & GaK-1228 & 2060 & 100 & - & $1710-2300$ & Charcoal & - & Zone 2,30 inches bs & Birks and Birks (1978) \\
\hline Viti Levu & Yanuca & GaK-1229 & Modern & - & - & - & Charcoal & - & Zone 2,10 inches bs & Birks and Birks (1978) \\
\hline Viti Levu & Karobo & ANU-10780 & 1780 & 80 & $-24 \pm 2.0 \mathrm{E}$ & $1420-1830$ & Charcoal & - & Square Ab-1: L7, $122 \mathrm{cmbs}$ & ANU-EPF \\
\hline Viti Levu & Karobo & ANU-10781 & 300 & 70 & $-24 \pm 2.0 \mathrm{E}$ & $0-500$ & Charcoal & - & Square Y: L3, $33 \mathrm{cmbs}$ & ANU-EPF \\
\hline Viti Levu & Karobo & ANU-11067 & 1680 & 70 & $-24 \pm 2.0 \mathrm{E}$ & $1370-1700$ & Charcoal & - & Square A2: L5, $100 \mathrm{cmbs}$ & ANU-EPF \\
\hline Viti Levu & Karobo & ANU-11068 & 2130 & 120 & $-24 \pm 2.0 \mathrm{E}$ & $1740-2340$ & Wood & cf. Cocos nucifera & Square A2: L5, $137 \mathrm{cmbs}$ & ANU-EPF \\
\hline Wakaya & Delaini & Beta 45789 & 300 & 70 & -26.0 & $0-490$ & Charcoal & - & Unit 2-D4, $40 \mathrm{cmbs}$ & Rechtman (1992: 202) \\
\hline Wakaya & Delaini & Beta 46438 & 140 & 50 & -17.5 & $0-130$ & Bone & ?Species & Unit 3-C3, $120 \mathrm{cmbs}$ & Rechtman (1992: 202) \\
\hline Wakaya & Delaini & Beta 46439 & 410 & 60 & -26.5 & $310-510$ & Charcoal & - & Unit 2-D4, $60 \mathrm{cmbs}$ & Rechtman (1992: 202) \\
\hline Wakaya & Delaini & Beta 46440 & 190 & 70 & -25.5 & $0-300$ & Charcoal & - & Unit 3-C3, $100 \mathrm{cmbs}$ & Rechtman (1992: 202) \\
\hline Wakaya & Delaini & Beta 46441 & 210 & 50 & -26.6 & $0-310$ & Charcoal & - & Unit 3-B4, $52 \mathrm{cmbs}$ & Rechtman (1992: 202) \\
\hline Wakaya & Delaini & Beta 46442 & 350 & 80 & -28.7 & $150-520$ & Charcoal & - & Unit 2-D2, $50 \mathrm{cmbs}$ & Rechtman (1992: 202) \\
\hline Wakaya & Delaini & Beta 46444 & 210 & 50 & -27.8 & $0-310$ & Charcoal & - & Unit 4-c4, 80 cmbs & Rechtman (1992: 202) \\
\hline
\end{tabular}


Table 19 continued

\begin{tabular}{|c|c|c|c|c|c|c|c|c|c|c|}
\hline Island & Site & Lab Code & CRA & CRA SE & C13 & Cal. BP & $\begin{array}{l}\text { Sample } \\
\text { Type }\end{array}$ & Identification & Context & Reference \\
\hline Wakaya & Delaini & Beta 46445 & Modern & - & -26.2 & - & Charcoal & & Unit EP-5, depth $40 \mathrm{cmbs}$ & Rechtman (1992: 202) \\
\hline Wakaya & Delaini & Beta 46446 & 120 & 50 & -24.9 & $0-280$ & Charcoal & - & Unit 4- $\mathrm{C} 4,70 \mathrm{cmbs}$ & Rechtman (1992: 202) \\
\hline Wakaya & Delaini & Beta 49824 & 420 & 90 & -18.5 & $0-430$ & Bone & ?Species & Unit 2-C3,30 cmbs & Rechtman (1992: 202) \\
\hline Wakaya & Delaini & Beta 49825 & 40 & 50 & -22 & - & Bone & & Unit EP-11, depth $30 \mathrm{cmbs}$ & Rechtman (1992: 202) \\
\hline Wakaya & Delaini & Beta 49826 & 120 & 50 & -18.3 & $0-60$ & Bone & ?Species & Unit $3-(3,70 \mathrm{cmbs}$ & Rechtman (1992: 202) \\
\hline Wakaya & Korolevu A & Beta 45785 & 610 & 60 & -24.3 & $510-650$ & Charcoal & - & Unit 1, $60 \mathrm{cmbs}$ & Rechtman (1992: 202) \\
\hline Wakaya & Korolevu A & Beta 45786 & 440 & 60 & -25.8 & $320-530$ & Charcoal & - & Unit 2, $85 \mathrm{cmbs}$ & Rechtman (1992: 202) \\
\hline Wakaya & Korolevu A & Beta 45787 & 410 & 60 & -25.7 & $310-510$ & Charcoal & - & Unit $3,85 \mathrm{cmbs}$ & Rechtman (1992: 202) \\
\hline Wakaya & Korolevu A & Beta 45790 & 540 & 80 & -19.0 & $150-510$ & Bone & ?Species & Unit 5, $40 \mathrm{cmbs}$ & Rechtman (1992: 202) \\
\hline Wakaya & Korolevu A & Beta 49827 & 60 & 50 & 3.3 & - & Marine shell & ?Species & Unit EPA, $20 \mathrm{cmbs}$ & Rechtman (1992: 202) \\
\hline Wakaya & Korolevu A & Beta 49828 & 160 & 50 & 3.4 & - & Marine shell & ?Species & Unit EPA, $20 \mathrm{cmbs}$ & Rechtman (1992: 202) \\
\hline Wakaya & Korolevu B & Beta 45788 & 80 & 60 & -27.3 & - & Charcoal & - & Unit 2a, $36 \mathrm{cmbs}$ & Rechtman (1992: 202) \\
\hline Wakaya & Korolevu B & Beta 45791 & 190 & 60 & -18.9 & $0-240$ & Bone & ?Species & Unit 1, $100 \mathrm{cmbs}$ & Rechtman (1992: 202) \\
\hline Wakaya & Korolevu B & Beta 46443 & 1950 & 70 & -27.7 & $1630-2000$ & Charcoal & & Unit 5, $122 \mathrm{cmbs}$ & Rechtman (1992: 202) \\
\hline Wakaya & Korolevu B & Beta 50522 & 40 & 70 & -19.6 & - & Bone & ?Species & Unit 5, $35 \mathrm{cmbs}$ & Rechtman (1992: 202) \\
\hline Wakaya & Korolevu B & Beta 50523 & 210 & 80 & -20.2 & $0-260$ & Bone & ?Species & Unit 1, $20 \mathrm{cmbs}$ & Rechtman (1992: 202) \\
\hline Yacata & Nadrodrodro & ANU-10811 & 1040 & 90 & $-24.0 \mathrm{E}$ & $690-1080$ & Charcoal & - & Layer 2: 52-60 cmbs & Clark and Hope (2001) \\
\hline Yadua & Vagairiki-1 & Wk-15423 & 870 & 30 & $3.1 \pm 0.2$ & $440-540$ & Marine shell & Trochus niloticus & Pit T1:130-140 cmbs & Nunn et al. (2004) \\
\hline Yadua & Vagairiki-2 & Wk-15424 & 760 & 40 & $3.3 \pm 0.2$ & $300-480$ & Marine shell & Trochus niloticus & Pit T1:140-150 cmbs & Nunn et al. (2004) \\
\hline Yadua & Denimanu & Wk-15425 & 2427 & 40 & $-26.0 \pm 0.2$ & $2320-2700$ & Charcoal & - & Pit T3:120-130 cmbs & Nunn et al. (2004) \\
\hline Yasawa & Y1-12 & AA-60257 & 156 & 30 & -24.2 & $0-280$ & Charcoal & - & TU1, L.I, Ivl. 2 & Cochrane (2004: 191) \\
\hline Yasawa & Y1-15 & AA- 60255 & 2207 & 40 & -25.6 & $2000-2310$ & $\begin{array}{l}\text { Carbonised } \\
\text { residue }\end{array}$ & - & TU 5, L.III, Ivl. 14 & Cochrane (2004: 191) \\
\hline Yasawa & Y1-15 & AA- 60256 & 607 & 30 & -27.1 & $520-630$ & $\begin{array}{l}\text { Carbonised } \\
\text { residue }\end{array}$ & - & TU 5, L.I, Ivl. 7 & Cochrane (2004: 191) \\
\hline Yasawa & $Y 2-22$ & Wk-6482 & 500 & 50 & - & $0-240$ & Marine shell & Trochus sp. & Surface & Cochrane (2004: 191) \\
\hline Yasawa & $Y 2-25$ & Beta-86839 & 2540 & 50 & -28.2 & $2360-2730$ & Charcoal & - & TU3, L.II base & Cochrane (2004: 191) \\
\hline Yasawa & $Y 2-25$ & Beta- 86840 & 2570 & 90 & -28.7 & $2360-2760$ & Charcoal & - & TU3, pit feature1 & Cochrane (2004: 191) \\
\hline Yasawa & Y2-25 & CAMS-24946 & 2530 & 50 & - & $2160-2650$ & Bone & Human & L.II & Cochrane (2004: 191) \\
\hline Yasawa & Y2-39 & Beta-174986 & 780 & 40 & -26.4 & $570-730$ & Charcoal & - & TU3, L.III & Cochrane (2004: 191) \\
\hline Yasawa & Y2-39 & Beta-52221 & 2260 & 90 & -28.2 & $1950-2430$ & Charcoal & - & TU1, L.IV, IVl. 22 & Cochrane (2004: 191) \\
\hline Yasawa & Y2-39 & Beta-53193 & 2790 & 260 & -28.0 & $2160-3470$ & Charcoal & - & TU1, L.IV, Ivl. 23 & Cochrane (2004: 191) \\
\hline Yasawa & Y2-39 & Beta-53194 & 2870 & 110 & -27.2 & $2740-3250$ & Charcoal & - & TU1, L.IV, IVl. 21 & Cochrane (2004: 191) \\
\hline Yasawa & Y2-39 & Beta-53195 & 2400 & 80 & -27.2 & $2160-2710$ & Charcoal & - & TU1, L.IV, Ivl. 17 & Cochrane (2004: 191) \\
\hline Yasawa & Y2-39 & Beta-53196 & 1160 & 80 & -26.6 & $820-1260$ & Charcoal & - & TU1, L.III, Ix|. 12 & Cochrane (2004: 191) \\
\hline Yasawa & Y2-39 & Beta-53197 & 330 & 70 & -27.4 & $150-500$ & Charcoal & - & TU1, L.II, ivl 6 & Cochrane (2004: 191) \\
\hline Yasawa & Y2-45 & Wk-6485 & 480 & 50 & - & $0-230$ & Marine shell & Trochus sp. & Surface & Cochrane (2004: 191) \\
\hline Yasawa & Y2-46 & Beta-93971 & 360 & 90 & -25.7 & $0-530$ & Charcoal & - & TU1, L.I, Ivl. 1 & Cochrane (2004: 191) \\
\hline
\end{tabular}




\section{Kulu Bay}

The Lapita site at Kulu Bay is in swampy terrain behind a low beach ridge that had been affected by stream cutting and mixing of beach materials with a fine, sticky clay-soil from nearby hill slopes. The six radiocarbon determinations clearly demonstrate extensive site disturbance, as three charcoal samples have a modern or recent age regardless of sample depth (ANU-10743, ANU-107949, ANU-107950), and one result on charcoal has a recent age of 560-910 (ANU-10727) that is too recent considering the presence of early pottery. Results indicate substantial mobilisation and deposition of slope sediments on to the Kulu beach flat in the last millennium. In contrast, two marine-shell dates with identical results of 2120-2380 cal. BP (Beta-107947, Beta-107948) indicate the redeposition of cultural material of Lapita and postLapita age in the stream-affected back-beach deposits.

\section{Karobo}

Adequate provenance information was available for one wood and three charcoal samples and these were submitted to the QDRC (Table 17). The first sample listed was collected from the base of Layer 3 in Square Y, and dated an earth oven (ANU-10781). The excavation notes mention the possibility that Square Y ceramics were not associated with the oven as most were found below Layer 3. The recent age of the oven (0-500 cal. BP) suggests this was probably the case. The remaining two determinations on charcoal came from a ceramic 'horizon' where Palmer and Golson had recorded abundant remains of flat-bottomed dishes, relief-marked ceramics and oven stones. The dates range from 1370 to $1830 \mathrm{cal}$. BP (ANU-10780, ANU-11067), similar to Level 2 determinations from Sigatoka where cognate ceramics have been recorded (Birks 1973; Burley 1997:Table 2).

The fourth date (ANU-11068) from Layer 5 is older and barely overlaps with charcoal determinations from the same layer $(1740-2340 \mathrm{cal}$. BP). The sample is a rectangular piece of cut palm wood, thought to be coconut (Palmer 1965:56). At Karobo, the excavators recorded no evidence for an occupation below the 'horizon' containing platter fragments, lenses of charcoal and oven stones.

Introduction of the worked palm wood might have occurred at an earlier time during the formation of the sand ridge, perhaps by flooding and channel cutting of the sand ridge by swamp water. Alternatively, the date itself might be questioned in view of the difficult pre-treatment required to extract cellulose from wood samples (A. Alimanovic, pers. comm., QDRC).

\section{Malaqereqere}

Three determinations on charcoal from the highly stratified cultural sediments in the Malaqereqere rock shelter gave consistent age-depth results. The shelter is dated by ANU-10453 immediately above the limestone floor with an age of 560-910 cal. BP, and a date in the overlying level 10-20 cm below the surface extending to $330 \mathrm{cal}$. BP (ANU-10452). The stratigraphy and radiocarbon dates show that the relatively small and low shelter was first used late in prehistory around $750 \mathrm{cal}$. BP and was regularly visited from then on.

\section{Volivoli II and III}

The excavation in the narrow chamber of Volivoli II produced three samples of charcoal, which were dated. Two dates on samples from $30-40 \mathrm{~cm}$ and $50-60 \mathrm{~cm}$ were similar and had a range of 580-1300 ca. BP (ANU-10449, ANU-10451), while the other from 40-50 cm was older 1630-2040 cal. BP. (ANU-10450). It appears that despite the relatively clear stratigraphy 
recorded in excavation, the cultural deposit in the enclosed shelter space has been disturbed significantly.

Similar to other rock shelters, like Malaqereqere, Qaranioso I and II, and Tuvu, three age results from the Volivoli III site point to human use in the period 1200-1000 cal. BP. Two dates have almost identical CRAs and span 740-1170 cal. BP, although the charcoal was collected from 80-90 cm and 50-60 cm respectively (ANU-11019, ANU-11016), indicating either rapid deposit build-up, or the mixing of archaeological charcoals with older and younger deposits. The remaining result on charcoal collected from $20-30 \mathrm{~cm}$ depth had an age of $0-460 \mathrm{cal}$. BP (ANU-11018).

\section{Tuvu}

A single result on charcoal from a thin lens in Spit IV $(30-40 \mathrm{~cm})$ returned a calibrated age of 1180-1700 cal. BP (ANU-11020), with the large standard error of \pm 100 responsible for the substantial age range. The single date suggests people were using rock shelters in the middle reaches of the Sigatoka Valley towards the middle of the first millennium AD.

\section{Qaranioso I and II}

At the large cave known as Qaranioso I, a single ${ }^{14} \mathrm{C}$ date was obtained on charcoal collected from a small hearth at $30 \mathrm{~cm}$ depth. The result (ANU-11015) was an age of 920-1350 cal. BP. Excavation in the nearby but smaller cave called Qaranioso II revealed paddle-impressed and shell-stamped pottery in Layer $2(14-49 \mathrm{~cm})$, with bone of the extinct giant iguana in Layer $3(50-65 \mathrm{~cm})$. Giant-iguana bone was submitted for ${ }^{14} \mathrm{C}$ AMS dating to the Oxford University Radiocarbon Accelerator Unit. However, it proved to contain too little collagen for dating. A single result on charcoal from $30 \mathrm{~cm}$ depth had a recent age of 530-670 cal. BP (ANU-11014).

\section{Natunuku}

Excavations at Natunuku were in a disturbed area where early and late prehistoric remains had been mixed together in the surface zone of the coastal site. Species and context information for three samples (ANU-11305, ANU-11306, ANU-11307) was misplaced, and a sample origin in Trench 3 is the only provenance information available. Two dates on charcoal were modern (ANU-10382, ANU-10381), while six marine-shell determinations were prehistoric, similar to Ugaga, where ovens had introduced recent charcoal into the deposit, and the marine-shell ages were prehistoric. The oldest shell dates (ANU-11305, ANU-10698, ANU-11307) extend to the terminal phase of the Lapita era, but no determinations appear to date the earliest cultural remains reported from Natunuku (Davidson et al. 1990; Davidson and Leach 1993), which were encountered by us only in displaced contexts. The other three marine-shell results have a span of 160-890 cal. BP (ANU-10699, ANU-10700, ANU-11306). Pottery from the site is stylistically varied, with diagnostic sherds from all periods of the sequence.

\section{Navatu 17A}

Six charcoal and three marine-shell samples from Trench B, and one charcoal sample from Trench A were dated at the QDRC. Radiocarbon results given in Table 17 have a consistent age-with-depth relationship. Median dates from the upper Layer 1 indicate an age of 150-500 cal. BP (ANU-10385, ANU-10710, ANU-10389). Layer 2 has an age spread of approximately 480-910 cal. BP (ANU-10709, ANU-10384). Basal dates on charcoal and marine shell from Layer 4 indicate deposition between 1360 and 1710 cal. BP (ANU-10708, ANU-10386), but 
two charcoal dates from levels slightly above these dates are more recent and overlap with the Layer 2 determinations. These dates (ANU-10390, ANU-10387) are from the eastern B1+2 squares, while the earlier dates are from the down-slope B3+4 squares. It appears that cultural remains from the oldest Layer 4 occupation, dating to ca. $1500 \mathrm{cal}$. BP, were mixed, at least in the $\mathrm{B} 1+2$ area, with more recent remains with an age of $660-1350 \mathrm{cal}$. BP.

\section{Thermoluminescence results}

Six pot sherds and samples of surrounding soil were sent to Gillian Robertson (Department of Physics, University of Adelaide). Two plain sherds were found to be suitable for dating with the fine-grain thermoluminescence (TL) technique. Dose rates from radioactive elements in the sherds and surrounding soils were calculated from thick-source alpha counting of uranium and thorium, and from X-ray spectrometer analysis of potassium (Table 18). A contribution from cosmic rays was included in the calculation of total dose. The uranium and thorium concentrations are very low, as expected from previous experiences with South Pacific pottery. The potassium levels are also similar to other Fijian samples (Prescott et al. 1982). In calculating the dose rate it is necessary to take into account the water content of the site. At Navatu the samples were high in water content (22-24\%). A decrease in water content would increase the dose rate, and thus the estimated age could be lower by a few percent than that quoted.

Even so, the TL ages appear too old compared with calibrated marine-shell and charcoal radiocarbon ages from similar contexts which do not overlap at two standard deviations. The discrepancy between the two dating techniques could be caused by the small quantity of quartz often present in Navatu and Fijian ceramics (Dickinson 1971; Prescott et al. 1982), and uncertainty over the water content of the Navatu 17A deposits during the period of burial. Whatever the reason for the discrepancy, the radiocarbon results are considered the more reliable age estimates.

\section{Votua}

Eight radiocarbon determinations on marine shell and charcoal from the 1996 excavations at Votua were reported in Clark et al. (2001), and another two dates were obtained on samples from Area 2 excavated in 2000. Table 17 lists the full set of Votua age results, which comprises seven marine shell and three charcoal dates. Determinations were on two shellfish species with different environmental preferences - with Anadara antiquata a common inhabitant of intertidal coral sand and Trochus niloticus associated with intertidal reef flats, and on unidentified wood charcoal. Seven ${ }^{14} \mathrm{C}$ ages indicate Lapita occupation at Votua between 2650 and $2800 \mathrm{cal}$. BP.

Two determinations have longer spans, with lower ranges extending to the period 2170 2470 cal. BP, but ANU-10706 on charcoal has a large standard error ( \pm 120 years), while ANU11527 on Anadara intercepts a plateau on the marine calibration curve (Stuiver and Braziunas 1993:Figure 17F). Neither provides reliable evidence that occupation at Votua lasted several centuries. A third date on Trochus niloticus (ANU-11069A) has an age of 1900-2300 cal. BP. The sample came from within the Area 1 shell midden and is suspected to have been contaminated by younger carbonates that were not removed by the pretreatment process (A. Alimanovic, pers. comm., QDRC). The difference is not related to the effects of dietary variation between species because a 'B' sample of Trochus shell from the spit above ANU-11069A gave a CRA of 2990 \pm 60 BP (ANU-11069B).

The pooled mean for six marine-shell dates was $2940 \mathrm{BP}$, which has a calibrated range of 2680-2760 cal. BP. Two charcoal ages have a pooled mean of $2690 \mathrm{BP}$, and an age span of 2690-2850 cal. BP ( $\mathrm{p}=0.83$ ), which is almost identical to the calibrated age of the pooled marine-shell age. 


\section{Sovanibeka}

There are three radiocarbon dates for Sovanibeka on three different types of material. The first was on a sample of Anadara antiquata taken from a bulk sample of Test Pit 2 material collected at 30-50 cm depth, which also produced a dentate-stamped sherd. This date (ANU-10779) has a calibrated age of 2360-2720 cal. BP, which overlaps with determinations from the nearby Votua site. The association of the shell sample with Lapita materials is unclear from the context and it might indicate later use of the cave after abandonment of the Votua site.

The second determination (ANU-11246) was on land-snail shell (Gonatorhaphe lavensis) from a Test Pit 1 bulk sample at the interface between an upper cultural-material/rat-bone deposit and the lower deposit of red silt with abundant land snails. The small number of land snails in the upper sediment suggests either that human activity impacted the local vegetation, or that the introduction of rats (Rattus praetor and Rattus exulans) in prehistory caused a decline in the number of land snails.

If the land-snail result was post-Lapita in age it might suggest the Votua occupation caused only minor vegetation modification in areas close to the Lapita occupation. Alternatively, an age result coterminous with Lapita settlement might indicate that widespread vegetation change occurred during the phase of initial settlement and extended beyond the immediate zone of occupation, indicating widespread clearing.

The sample of land-snail shell gave a CRA of $4290 \pm 60 \mathrm{BP}$, which does not provide a useful estimate for dating human impact on local vegetation. It is feasible that the age is too old because land snails living in the limestone chamber were absorbing old carbon in their diet. Dating of modern and prehistoric land snails suggests that species living in limestone areas are more likely to absorb old carbon than those living in non-limestone areas (e.g. Rafter et al. 1972; Goodfriend and Stipp 1983). The explanation for the early land-snail age advanced here is that either prehistoric human and rat activity in the chamber caused sediment compaction and mixing of cultural remains with those of land snails deposited in the mid-Holocene, or the land snails were absorbing old carbon from limestone.

The third determination was on a purified bone collagen from a rat humerus identified as Rattus praetor, the large spiny rat (K. Aplin, pers. comm., CSIRO). The humerus was recovered from Test Pit 1 at 50-60 cm depth in the transition zone between sediments containing cultural material and the underlying natural deposit. Rat bone in the size range of Rattus praetor was also found in the Votua excavations, but two samples submitted to ANSTO had insufficient collagen after pretreatment to obtain an AMS date. The calibrated result for OZF882 of 670-770 cal. $\mathrm{BP}$ is considerably later than anticipated and, if accurate, might suggest that Rattus praetor was a relatively late prehistoric arrival to Fiji, or that it was introduced in the Lapita phase and survived until the arrival of European rats in the early 19th century. An attempt to clarify whether rat arrival was coincident with Lapita expansion by AMS dating of two Rattus praetor bones recovered from the basal levels of the Navaprah site in Vanuatu (Bedford 2000) also failed due to low collagen yields. In short, although Rattus praetor remains have been identified in New Ireland, Reef/Santa Cruz Islands, Tikopia, Vanuatu and Fiji (White et al. 2000), it is unclear when the species first arrived in Fiji, how long it persisted, what impact the rat had on native fauna and flora, and when it became extinct.

\section{Review of radiocarbon dates from Fiji}

A goal of the EPF dating program was to establish whether evidence of human occupation older than Lapita culture could be identified in open sites and caves/rock shelters, and if it could not, to accurately date the Lapita phase in Fiji. A second objective examined the change from 
Lapita-influenced ceramic assemblages to those with a distinctive Fijian character during the first millennium $\mathrm{AD}$. The stylistic boundary between Lapita and post-Lapita pottery assemblages has been viewed as a formative period when traits characteristic of the late prehistoric period emerged in nascent form. These include an explicit concern with defence and territory, manifested by the development of constructed fortifications, cannibalism, agricultural intensification, craft specialisation and the movement of populations inland.

These topics have been to the forefront of archaeological research in the archipelago. They were first set out as three distinct issues in the 1960s by Bruce Palmer, Director of the Fiji Museum, and they continue to be important to prehistorians working in the Central Pacific. This is illustrated by considering the population of radiocarbon dates recovered from Fijian archaeological sites since the 1950 s, a list compiled by recording the ${ }^{14} \mathrm{C}$ ages listed in theses, reports and publications. We recorded all results, including modern determinations, ages which have large standard errors, and dates on material of potentially high inbuilt age such as turtle bone, and have not evaluated the cultural association of the analysed samples, an issue we return to in our discussion of the Fiji radiocarbon sequence. Determinations on bulk palaeosol humin were omitted, as sediment antiquity may be different from the age of cultural material found in it.

The results reported in Table 19 include the majority of ${ }^{14} \mathrm{C}$ determinations from Fiji, and although a few results have no doubt been missed, the dates demonstrate a continuing concern with major aspects of Bruce Palmer's research agenda. When examined by geographic location, most of the CRAs are from the large island of Viti Levu. Elsewhere, there is a reasonable number of determinations from the Yasawa Islands, Taveuni and parts of the Lau Group. Island size is not correlated with the amount of research activity, and there are no age results from Vanua Levu and Kandavu, respectively the second and third-equal largest landmasses, or from substantial islands such as Vatulele. Within Viti Levu most determinations are from the southwest coast area where several Lapita and mid-sequence ceramics sites, in addition to fortifications in the Sigatoka Valley, were recorded by Palmer $(1965,1966,1967,1969)$. An absolute chronology for human occupation of the interior of large islands of Fiji in prehistory has received little attention, with the exception of Field's (2004) study of fortifications in the Sigatoka Valley.

Thesis and published research also divide neatly into Palmer's three research subjects, with the study of warfare, fortifications and socio-political complexity (Frost 1970; Clunie 1977; Best 1984; Parry 1987; Rechtman 1992; Cochrane 2004; Field 2004), mid-sequence ceramic and social change (Hunt 1980; Best 1984; DeGusta 1999; Clark 2000; Burley 2003, 2005), and the excavation and analysis of Lapita sites (Birks 1973; Mead et al. 1973; Best 1981, 1984; Kay 1984; Anderson and Clark 1999, Clark and Anderson 2001a). The radiocarbon results from the EPF, along with age determinations obtained in recent investigations, now make it practicable to revisit the chronology of these recognised inflection points in the Fijian sequence. Calibrated ages at 2SD for the ${ }^{14} \mathrm{C}$ results listed in Table 19 are shown in Figure 84. Excluded dates were those with a standard error larger than \pm 100 years, 'modern' results, CRAs with an age less than $110 \mathrm{BP}$, and land-snail shell dates. Removing these leaves a total of 281 radiocarbon results.

\section{Lapita settlement of Fiji}

The chronology of Lapita expansion is controlled at the regional level by the age of the oldest site on an island or archipelago, since colonising populations are by definition inherently mobile, and uninhabited landscapes, even those relatively poor in natural food resources, appear to offer few impediments to the spread of Neolithic populations (Clark et al. 2006). Kirch and Hunt (1988:24) argued that 'frequency distributions for dates from the eastern and westernmost 
174 Geoffrey Clark and Atholl Anderson

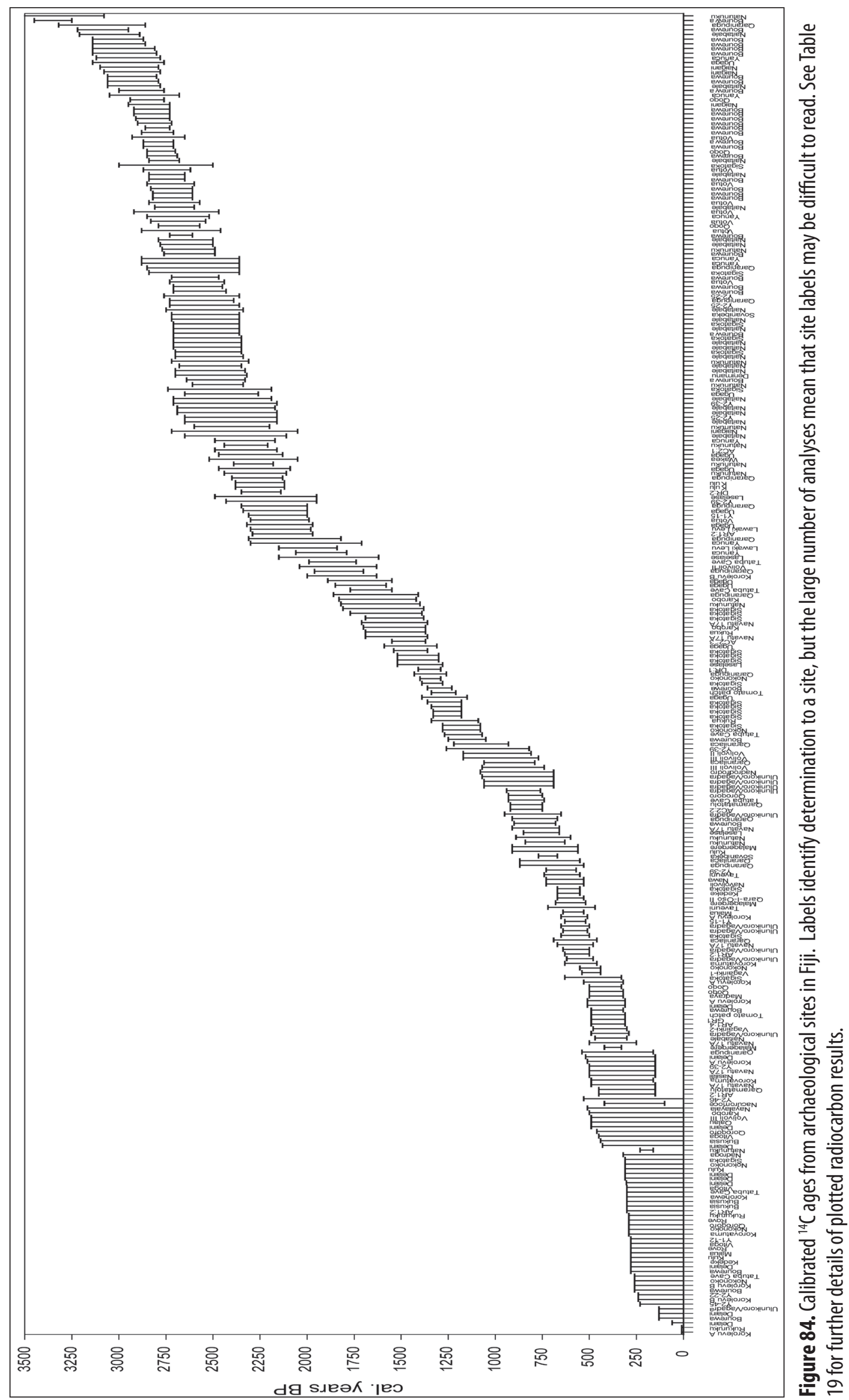


regions (Bismarcks and Fiji-Western Polynesia) displayed nearly identical ranges and modes', and that the Fijian radiocarbon ages on Lapita sites extended to about $3500 \mathrm{cal}$. BP. Spriggs (1990:19-20) in a critical review of available dates suggested that Fiji-West Polynesia was not occupied earlier than 3050 cal. BP, with the colonisation of Fiji later revised to 2800-2900 cal. BP from a survey of screened dates from Lapita sites (Anderson and Clark 1999).

The trend towards a more recent colonisation age reflected in the literature from the $1980 \mathrm{~s}$ to the end of the 1990s began almost immediately to reverse, with the Yanuca Lapita site dated to $2950 \mathrm{cal}$. BP or older (Clark and Anderson 2001b), and reports that the newly discovered Lapita sites of Bourwea and Naitabale might date to 3220 cal. BP (Kumar et al. 2004; Nunn et al. 2004b). Such claims were given support by the revision of the age of the Nukuleka (TO.2) site in Tonga to ca. 2900 cal. BP (Burley and Dickinson 2001), and the possibility that the Teouma site in Vanuatu dated to 3200-3000 cal. BP (Bedford et al. 2006). The revised colonisation age for Fiji and neighbouring island groups to the east and west was intriguing, as the age of Lapita sites in the Bismarck Archipelago was previously argued to be no older than 3300-3200 cal. BP (Specht and Gosden 1997). If Lapita occupation of Fiji began at $3200 \mathrm{cal}$. BP, it would imply a very rapid spread of Lapita groups eastward to Vanuatu and Fiji by $3200 \mathrm{cal}$. BP, followed by a substantial and puzzling hiatus of around 200-300 years before settlement of New Caledonia and Tonga at 3000-2900 cal. BP (Sand 1997; Burley and Dickinson 2001).

Inspection of the oldest ${ }^{14} \mathrm{C}$ results from $\mathrm{Fiji}$ has determinations from eight locations with calibrated spans extending to $3000 \mathrm{cal}$. BP or older. Rejected from this set are Gak-1218

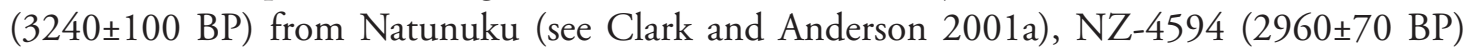

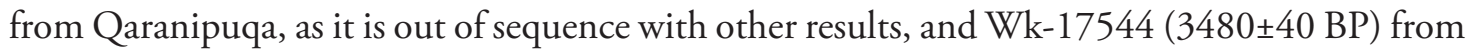
Bourewa which is significantly older than all other age results from the site. Single determinations on marine shell and charcoal from Ugaga Island, Sigatoka and Naitabale (Beta-107953,

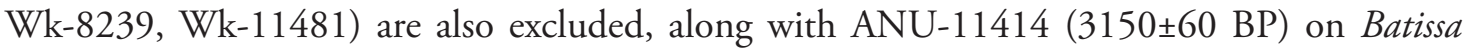
violacea, a freshwater shellfish that may incorporate old carbon from dissolved limestone in its shell. The last five dates may in fact be accurate determinations, but individual results on material known to produce unreliable results, and samples of uncertain cultural association, are inadequate to establish the antiquity of human colonisation.

Remaining dates are ANU-11417 (3050 \pm 60 BP) from Yanuca on Cyprea tigris, excavated by Birks and Birks (1978), with a calibrated age span of 2680-3050 cal. BP, and four marineshell dates from Naigani with a pooled CRA of 3110 BP, and a calibrated age of 2790-2980 cal. BP. The results suggest human arrival in the period ca. 3000-2900 cal. BP. Evidence for older occupation has recently been recorded from Bourewa, where there are eight dates indicating Lapita occupation in Fiji began before $3000 \mathrm{cal}$. BP. These include paired dates on charcoal from Unit 3 (Wk-14235, Wk-14236) and Unit X3 (Wk-17542, Wk-17937), and paired charcoalmarine shell results from Unit 4 (Wk-14237, Wk-14595). Although two early ages on marine shell were on small species (Tellinidae sp. and Atacodea striata) that are often found naturally deposited on beaches, the oldest CRA (Wk-14237) from Unit 4 is supported by Wk-14595 on charcoal from the same level. The six oldest charcoal determinations from Bourewa have a pooled CRA of 2894 BP and a 2SD age span of 2870-3080 cal. BP. The two oldest marine-shell dates have a pooled CRA of $3180 \mathrm{BP}$, and an almost identical range to the combined charcoal result of 2870-3090 cal. BP. A conservative interpretation of these dates, considering that the charcoal has not been identified to taxa, and the possibility that some marine shellfish have been wave deposited, suggests Lapita colonisation of Fiji in the period 2950-3050 cal. BP, rather than 3200 cal. BP. 


\section{Mid-sequence ceramic change}

The three sites where some of the most important mid-sequence deposits in Fiji have been found - Sigatoka VL 16/1, Yanuca VL 16/81 and Natunuku VL 1/1 - were excavated initially because of the discovery of Lapita ceramics, and the mid-sequence materials recovered were used only to aid the establishment of a ceramic sequence. Initially described by Green (1963) as the Navatu phase (2100-900 BP), the mid-sequence was distinguished by a high proportion of relief-marked sherds (up to $50 \%$ ), including cross-hatch or diamond relief, parallel rib and 'wavy' impressed sherds, with the proportion of relief-marked pottery declining by the end of the phase to approximately $20 \%$ of the assemblage.

Differences between Lapita and mid-sequence ceramics in vessel form and decoration were linked by Palmer (1965:33) to the Sa-Huynh-Kalanay ceramics of Southeast Asia. The makers of relief pottery were therefore identified as proto-Polynesians. Previously, Gifford and Shutler (1956) had noted the similarity between parallel-rib and cross-hatch relief sherds from New Caledonia and sherds from Fiji, and Garanger (1971:58) linked cord-impressed ceramics found on the Mele Plain on Efate Island in Vanuatu with two Fijian sherds impressed with woven fibre and excavated from Navatu 17A (Gifford 1951a). However, recent work has shown that the Mele Plain sherds were made in Japan and belong to the early Jomon tradition (Dickinson et al. 1999). Until the past decade, there have been few well-dated archaeological sites in Fiji, New Caledonia and Vanuatu with which to compare the antiquity of widespread decorative traits, and examine the similarities and differences among regional ceramic sequences.

A chronology of mid-sequence ceramic change was first proposed by Best (1984), who identified two points of stylistic change in Lakeba ceramics, which he interpreted as evidence for population movements from New Caledonia and Vanuatu. The first, emanating from New Caledonia at ca. $2100 \mathrm{cal}$. BP, introduced an ovoid vessel with sharply everted rims and square lips that was decorated with parallel-rib or cross-hatch relief markings. The second, at $1700 \mathrm{cal}$. BP, correlated with the appearance of minor decorative techniques such as asymmetric and fingernail incision, finger pinching, cord-wrapped paddle impressing and rim notching. These traits were seen as characteristic of Vanuatu ceramic traditions that had been introduced to Fiji.

Radiocarbon results from the EPF at the mid-sequence sites of Navatu 17A, Karobo and Ugaga also suggested a major ceramic change took place around 2300-1900 cal. BP, when the Lapita-derived ceramic trajectory was altered when jars and bowls marked with heavy parallelrelief impressions, dents and deep wiping appeared (Clark 2000). The dating of the transition was poorly focused, however, due to substantial site disturbance at Ugaga (Table 17), although the presence of mid-sequence ceramics dating to around $1500 \mathrm{cal}$. BP at Navatu 17A and Karobo indicated that ceramic change had occurred somewhat earlier.

Investigations at the Sigatoka Sand Dune by Burley $(2003,2005)$ revealed two distinct midsequence ceramic assemblages separated by a thin sand layer. The lower, termed 'Fijian Plainware', includes ovoid jars and decorative techniques of parallel and cross-hatch relief, punctate and lip notching. This assemblage is seen as having continuity in vessel form and technology with Lapita ceramics (Burley 2005:321). The upper ceramics, called 'Navatu', comprise globular jars with highly everted rims, lip notching, end-tool and side impressions, and fingernail pinching/ gouging (see Chapter 12).

The 'Navatu' deposit at Sigatoka was dated by three ${ }^{14} \mathrm{C}$ results (CAMS-70090, CAMS-70091, CAMS-70920), with a pooled CRA of $1437 \mathrm{BP}$, calibrating to 1275-1340 cal. BP. Five results date the lower Fijian Plainware levels, with two determinations (CAMS-68195 and CAMS-70921) considered to be outliers (Burley 2005:325). The three oldest ${ }^{14} \mathrm{C}$ ages have a pooled age of $1570 \mathrm{BP}$, and an age range of 1335-1516 cal. BP, indicating a rapid transition 
to 'Navatu' pottery over a century. The presence of 'Navatu' pottery traits on Lakeba in east Fiji at 1700-1600 cal. BP, and the later arrival of these traits in north and south Viti Levu at about $1300 \mathrm{cal}$. BP is suggested to be the result of gradual east-to-west movement of 'Navatu'-related groups, or ceramic transmission from the Lau Group (Burley 2005:342). Additional examination of stylistic phases and ceramic transformation through ${ }^{14} \mathrm{C}$ dating mid-sequence deposits is particularly important for identifying the direction and extent of population movements within and beyond the Fiji Islands.

\section{Fortifications and defensive sites}

Studies have shown that the development of social complexity in Fiji was associated with the appearance of fortifications and horticultural intensification (Palmer 1969; Frost 1970; Parry 1987), with population growth and control over horticultural resources being key attributes that led to the 'classic' Fijian chiefdoms (Parry 1987; Field 2004). Establishing an absolute chronology for defensive sites provides a proxy for the broader socio-political and economic transformations in Fiji prehistory.

Radiocarbon dating of fortified sites suggests that most of those on Taveuni, Wakaya and Lakeba date to the past 700 years or less. On Taveuni, defensive sites like Navolivoli and Nawa were probably first used about 500-700 cal. BP (Gak-2411, Gak-2414), while those on Wakaya (Delaini and Korolevu) date slightly later to 500-300 cal. BP (Beta-45787, Beta-45790). The massive hill fort of Ulunikoro, located on a limestone plateau on Lakeba in the Lau Group, is older still, with CRAs on charcoal and marine shell (NZ-4044, NZ-4585, NZ-4586, NZ-4587, NZ-5180) indicating occupation at 800-1000 cal. BP. On Beqa Island, a very large fortification, also known as Korolevu, has been recorded, but according to Crosby (1988:236), it is probably less than 500 years old.

Fortifications, like most prehistoric structures built of earth and stone in the Central Pacific such as house mounds, tombs, walls and roads, are difficult to radiocarbon date because construction, use and reuse can incorporate samples of different ages in a structure. In addition, unmodified strategic locations used for defence and refuge are likely to have lower site visibility than sites associated with constructed defences. In a nuanced study of sites in the Sigatoka Valley, Field $(2004,2005)$ showed that natural defences such as large caves and remote peaks (Tatuba, Nokonoko) were used as territorial strongholds and refuges by $1400 \mathrm{cal}$. BP (AA-50287, AA-50283, AA-50298). The establishment of sites with constructed features on highly visible outcrops (Malua, Korovatuma) began at 500-600 cal. BP (AA-50295, AA-50300), with the emergence of new ring-ditch sites on the valley floor (Bukusia and Vitonga) at 150-350 cal. BP (AA-50309, AA-50302, AA-50304, Wk-11135).

The EPF dating program, while not aimed specifically at defensive sites, nonetheless investigated several rock shelters and deposits with a potential refuge/defensive aspect. Chief among these is the Navatu 17A site on the flanks of a volcanic plug, which was used as a refuge in the 19th century. The lowest cultural deposit included cannibalised human bone from the B3+4 square, and is dated to $1360-1710$ cal. BP. The site position is not strongly defensive, but might have been chosen due to its proximity to a high refuge. The presence of cannibalism at Navatu supports Field's hypothesis that inter-group conflict was becoming more prevalent, and as a result, defensive considerations began to influence site location and position in the period 1000-1500 cal. BP. Interestingly, this is the period when Burley $(2003,2005)$ identifies a significant mid-sequence change in the ceramics and economy at the Sigatoka Sand Dunes, and Dickinson (2006:117-119) records several examples of long-distance ceramic transfer from Fiji to islands outside the archipelago. 


\section{Conclusion}

The EPF resulted in reliable radiocarbon dates for the late-Lapita site at Votua, but at Natunuku, Ugaga and Kulu Bay, the antiquity of the oldest remains could not be determined due to redeposition and mixing of cultural material of different ages and the absence of dateable material at Qaranioso II. Deposits of mid-sequence age at Navatu 17A and Karobo were better delineated, and at Ugaga the majority of ${ }^{14} \mathrm{C}$ dates spanned $2500-1200 \mathrm{cal}$. BP, suggesting that many of the cultural remains from the island covered the late-Lapita to mid-sequence transition. Several rock shelters on Viti Levi (Malaqereqere, Volivoli II and III, Tuvu and Qaranioso II) were mainly used in the past 1000 years, with several of the cultural deposits including evidence for occasional use in late-Lapita (Qaranioso II) and mid-sequence (Volivoli II) times.

Combining the EPF determinations with those from recent and earlier studies showed that the earliest evidence for Lapita colonisation is at Bourewa in western Viti Levu, which is dated to 2950-3050 cal. BP. Other results from sites on Viti Levu and nearby islands such as Ugaga, Naigani, Yanuca and Naitabale suggest that Lapita groups were either highly mobile or relatively numerous, and both attributes may have been in play. While the size of the migrant population entering Fiji was sufficient to leave a presence on Viti Levu and nearby islands, the population does not appear to have reached the Lau Group until 2850-2900 cal. BP.

The intra-archipelago colonisation interval is significant, as it suggests that the presence of large islands like Viti Levu (10,947 sq. km) diverted human movement, for a time, away from long-distance expansion and instead towards coastal and inland exploration of a large island. One means of testing this hypothesis is to look at the southernmost islands of the Lau Group that are roughly equidistant from Viti Levu and Tonga. For instance, the ceramic and materialculture assemblages of Lapita sites on islands like those in the Ono-i-lau Group and Vatoa could show, when examined, a closer affinity to Tongan Lapita materials than to Lapita ceramics from western Fiji. Such a finding would be consistent with the independent colonisation of Tonga from a location like the Reef Santa Cruz Islands (Burley and Dickinson 2001), and the expansion of Lapita groups in Tonga to neighbouring and possibly unoccupied islands in the Lau Group. Similarly, if the oldest Lapita ceramics in southern Lau were shown to have an affinity with ceramics from other parts of Fiji, but dated 100-200 years later, it would indicate a noticeable decline in the dispersal rate of Lapita groups after human arrival in west Fiji. One outcome of such a finding would be that very different social and demographic conditions characterised the colonisation of west Fiji than the colonisation of east Fiji.

Dating the mid-sequence transition at the Sigatoka Sand Dunes indicates that stylistic change can occur rapidly over about century from ca. 1400-1300 cal. BP, which is significant, as the rate of ceramic change is frequently correlated in archaeology with major social change, particularly the arrival or influence of new populations (Hunt 1986). The ceramic record of the Fiji Islands, located on the ethnological border between Melanesia and Polynesia, has been scrutinised particularly for evidence of cultural intrusion (Clark 2003) because the people of Fiji have different physical characteristics from nearby populations in West Polynesia. It is important to note that while the rate of ceramic change appears to be rapid, radiocarbon dating of fortifications and habitation sites indicates that wider changes were occurring in Fijian society during the mid-sequence. These include the expansion of populations to the interior of large and small islands, the use of naturally defended locations, and the presence of cannibalism at ca. 1400 cal. BP. A significant episode of external population arrival and impact in Fiji has not been identified convincingly in the archaeological record, and several researchers have rejected migration as an explanation for the advent of fortifications, or the cause of stylistic change 
in pottery (Clark 2000; Cochrane 2004; Field 2004). This is an issue yet to be resolved (e.g. Chapter 12; Burley 2005).

The chronology of the Fiji sequence, although based on a relatively large number of ${ }^{14} \mathrm{C}$ results, has long focused on clarifying the temporal boundaries of three parts of the sequence (colonisation/mid-sequence/social complexity). As the above review shows, refinements to the absolute chronology have raised new questions about the pattern of Lapita dispersal, and have closed the gap between the timing of mid-sequence ceramic change and the advent of population expansion and territoriality, previously seen as distinct events separated by 500 or more years. Several major geographical and temporal gaps remain, with few intact assemblages from the interval $2500-1500 \mathrm{cal}$. BP adequately dated, and the possibility of regional variation in Fijian material culture still to be examined by the recovery of well-dated sequences from Vanua Levu and several other large islands that have yet to receive even cursory archaeological research. A number of recognised site types have also to be dated, such as the enigmatic naga enclosures (Palmer 1971), agricultural field systems (e.g. Kuhlken and Crosby 1999) and adze and flake quarries (see Chapter 14).

\section{References}

Anderson, A. and Clark, G. 1999. The age of Lapita settlement in Fiji. Archaeology in Oceania 34: 31-39.

Anderson, A., Roberts, R., Dickinson, W., Clark, G., Burley, D., De Biran, A., Hope, G. and Nunn, P. 2006. Times of sand: Sedimentary history and archaeology at the Sigatoka Dunes, Fiji. Geoarchaeology 21: 131-154.

Anderson, A., Clark, G. and Worthy, T. 2000. An inland Lapita site in Fiji. Journal of the Polynesian Society 109: 311-316.

Bedford, S.H. 2000. Pieces of the Vanuatu puzzle: Archaeology of the North, South and Centre. Unpublished PhD thesis, Australian National University, Canberra.

Bedford, S., Spriggs, M. and Regenvanu, R. 2006. The Teouma Lapita site and the early human settlement of the Pacific Islands. Antiquity 80: 812-828.

Best, S. 1981. Excavations at Site VL 21/5 Naigani Island, Fiji, a preliminary report. Department of Anthropology, University of Auckland.

Best, S. 1984. Lakeba: The prehistory of a Fijian Island. Unpublished PhD thesis, Department of Anthropology, University of Auckland.

Best, S. 1987. The Sigatoka dune burials (Site VL 16/1). Unpublished report to the Fiji Museum, Suva.

Birks, L. 1973. Archaeological excavations at Sigatoka dune site, Fiji. Bulletin of the Fiji Museum No.1.

Birks, L. and Birks, H. 1978. Archaeological excavations at site VL16/81, Yanuca Island, Fiji. Oceanic Prehistory Records No. 6. University of Auckland.

Burley, D. 1997. Archaeological research Sigatoka Sand Dune National Park. Unpublished report to the Fiji Museum, Suva.

Burley, D.V. 2003. Dynamic landscapes and episodic occupations: Archaeological interpretation and implications in the prehistory of the Sigatoka Sand Dunes. In: Sand, C. (ed), Pacific Archaeology: Assessments and prospects, pp. 307-315. Les Cahiers de l' Archéologie en Nouvelle-Calédonie Number 15, New Caledonia.

Burley, D.V. 2005. Mid-sequence archaeology at the Sigatoka Sand Dunes with interpretive implications for Fijian and oceanic culture history. Asian Perspectives 44: 320-348.

Burley, D.V. and Dickinson, W.R. 2001. Origin and significance of a founding settlement in Polynesia. Proceedings of the National Academy of Sciences of the USA 98: 11829-11831. 
Clark, G. 2000. Post-Lapita Fiji: Cultural transformation in the Mid-sequence. Unpublished PhD thesis, Australian National University, Canberra.

Clark, G. 2003. Shards of meaning: Archaeology and the Melanesia-Polynesia distinction. Journal of Pacific History 38: 197-213.

Clark, G. and Sorovi-Vunidilo, T. 1999. Fijian double-spouted vessels. Domodomo 11: 6-14.

Clark, G. and Anderson, A. 2001a. The pattern of Lapita settlement in Fiji. Archaeology in Oceania 36: 77-88.

Clark, G. and Anderson, A. 2001b. The age of the Yanuca Lapita site, Viti Levu, Fiji. New Zealand Journal of Archaeology 22: 15-30.

Clark, G., Anderson, A. and Matararaba, S. 2001. The Lapita site at Votua, northern Lau Islands, Fiji. Archaeology in Oceania 36: 134-145.

Clark, G and Hope, G. 2001. Archaeological and palaeoenvironmental investigations on Yacata Island, northern Lau, Fiji. Domodomo 13: 29-47.

Clark, G., Anderson, A. and Wright, D. 2006. Human colonization of the Palau Islands, western Micronesia. Journal of Island and Coastal Archaeology 1: 215-232.

Clark, J.T. and Cole, A.O. 1997. Environmental change and human prehistory in the Central Pacific: Archaeological and palynological investigations on Totoya Island, Fiji. Report to the Fiji Museum, Suva.

Clunie, F. 1977. Fijian weapons and warfare. Bulletin of the Fiji Museum No. 2.

Cochrane, E.C. 2004. Explaining cultural diversity in ancient Fiji: The transmission of ceramic variability. Unpublished PhD thesis, University of Hawaii.

Crosby, A. 1988. Beqa: archaeology, structure and history in Fiji. Unpublished MA thesis, Department of Anthropology, University of Auckland.

Davidson, J., Hinds, E., Holdaway, S. and Leach, F. 1990. The Lapita site of Natunuku, Fiji. New Zealand Journal of Archaeology 12: 121-155.

Davidson, J. and Leach, F. 1993. The chronology of the Natunuku site, Fiji. New Zealand Journal of Archaeology 15: 99-105.

De Biran, A. 2001. The Holocene geomorphic evolution of the Sigatoka Delta, Viti Levu, Fiji Islands. Unpublished PhD thesis, University of the South Pacific, Suva, Fiji Islands.

DeGusta, D. 1999. Fijian cannibalism: Osteological evidence from Navatu. American Journal of Physical Anthropology 110: 215-241.

Dickinson, W.R. 1971. Petrography of some sand tempers in prehistoric pottery from Viti Levu, Fiji. Final report No. 2. Records of the Fiji Museum 1: 108-121.

Dickinson, W.R. 2006. Temper sands in prehistoric Oceanian pottery: Geotectonics, sedimentology, petrography, provenance. The Geological Society of America Special Paper 406.

Dickinson, W.R., Burley, D.V., Nunn, P.D., Anderson, A., Hope, G., De Biran, A., Burke, C. and Matararaba, S. 1998. Geomorphic and archaeological landscapes of the Sigatoka Dune site, Viti Levu, Fiji: Interdisciplinary investigations. Asian Perspectives 37: 1-31.

Dickinson, W.R., Sinoto, Y. H., Shutler, R. Jr., Shutler, M.E., Garanger, J. and Teska, T.H. 1999. Japanese Jomon sherds in artifact collections from Mele Plain on Efate in Vanuatu. Archaeology in Oceania 34: 15-24.

Field, J.S. 2004. Environmental and climatic considerations: A hypothesis for conflict and the emergence of social complexity in Fijian prehistory. Journal of Anthropological Archaeology 23: 79-99.

Field, J.S. 2005. Land tenure, competition and ecology in Fijian prehistory. Antiquity 79: 586-600.

Frost, E.L. 1970. Archaeological excavations of fortified sites on Taveuni, Fiji. Unpublished PhD thesis, University of Oregon.

Frost, E.L. 1979. Fiji. In: Jennings, J.D. (ed), The prehistory of Polynesia, pp. 61-81. Australian National University Press.

Garanger, J. 1971. Incised and applied-relief pottery, its chronology and development in southeastern 
Melanesia, and extra areal comparisons. In: Green, R.C. and Kelly M. (eds), Studies in Oceanic Culture History volume 2, pp. 53-99. Pacific Anthropological Records No. 12.

Gifford, E.W. 1951a. Archaeological excavations in Fiji. University of California Anthropological Records 13: 189-288.

Gifford, E.W. 1951b. Six Fijian radiocarbon dates. Journal of the Polynesian Society 64: 240.

Gifford, E.W. and Shutler, D. Jr. 1956. Archaeological excavations in New Caledonia. Anthropological Records 18, No. 1. University of California Press, Berkeley and Los Angeles.

Green, R.C. 1963. A suggested revision of the Fiji sequence. Journal of the Polynesian Society 72: 235-253.

Goodfriend, G.A. and Stipp, J.J. 1983. Limestone and the problem of radiocarbon dating land-snail shell carbonate. Geology 11: 575-577.

Hughen, K.A., Baillie, M.G.L., Bard, E., Bayliss, A., Beck, J.W., Bertrand, C., Blackwell, P.G., Buck, C.E., Burr, G., Cutler, K.B., Damon, P.E., Edwards, R.L., Fairbanks, R.G., Friedrich, M., Guilderson, T.P., Kromer, B., McCormac, F.G., Manning, S., Bronk Ramsey, C., Reimer, P.J., Reimer, R.W., Remmele, S., Southon, J.R., Stuiver, M., Talamo, S., Taylor, F.W., Plicht, J. van der and Weyhenmeyer C.E. 2004. Marine04 marine radiocarbon age calibration 26-0 ka BP. Radiocarbon 46: 1059-1086.

Hunt, T.L. 1980. Toward Fiji's past; archaeological research on southwestern Viti Levu. Unpublished MA thesis, University of Auckland.

Hunt, T.L. 1986. Conceptual and substantive issues in Fijian prehistory. In: Kirch, P.V. (ed), Archaeological approaches to evolution and transformation, pp. 20-32. Cambridge University Press, Cambridge.

Jones, S., Steadman, D.W. and O'Day, P.M. 2007. Archaeological investigations on the Small Islands of Aiwa Levu and Aiwa Lailai, Lau Group Fiji. Journal of Island and Coastal Archaeology 2: 72-98.

Kay, R. 1984. Analysis of archaeological material from Naigani. Unpublished MA thesis, University of Auckland.

Kirch, P.V. and Hunt, T.L. 1988. The spatial and temporal boundaries of Lapita. In: Kirch, P.V. and Hunt, T.L. (eds), Archaeology of the Lapita cultural complex: A critical review, pp. 9-32. Thomas Burke Memorial Washington State Museum Research Report No. 5. Seattle: The Burke Museum.

Kuhlken, R. and Crosby, A. 1999. Agricultural terracing at Nakauvadra, Viti Levu: A late prehistoric irrigated agrosystem in Fiji. Asian Perspectives 38: 62-89.

Kumar, R., Nunn, P.D., Katayama, K., Oda, H., Matararaba, S. and Osborne, T. 2004. The earliestknown humans in Fiji and their pottery: The first dates from the 2002 excavations at Naitabale (Nartururuku), Moturiki Island. South Pacific Journal of Natural Science 22: 15-21.

Kumar, R., Nunn, P.D., Field, J. and De Biran, A. 2006. Human response to climate change around AD 1300: A case study of the Sigatoka Valley, Viti Levu Island, Fiji. Quaternary International 151: 133-143.

Leach, F., Quinn, C., Morrison, J. and Lyon, G. 2001. The use of multiple isotope signatures in reconstructing prehistoric human diet from archaeological bone from the Pacific and New Zealand. New Zealand Journal of Archaeology 23: 31-98.

McCormac, F.G., Hogg, A.G., Blackwell, P.G., Buck, C.E., Higham, T.F.G. and Reimer, P.J. 2004. SHCal04 Southern Hemisphere Calibration 0-11.0 cal kyr BP. Radiocarbon 46: 1087-1092.

Mead, S.M., Birks, L., Birks, H. and Shaw, E. 1973. The decorative system of the Lapita potters of Sigatoka, Fiji. Journal of the Polynesian Society Memoir Supplement 82: 1-43, 44-98.

Nunn, P.D., Matararaba, S., Ishimura, T., Kumar, R. and Nakoro, E. 2004a. Reconstructing the Lapita-era geography of Northern Fiji: A newly discovered Lapita site on Yadua Island and its implications. New Zealand Journal of Archaeology 26: 41-55.

Nunn, P.D., Kumar, R., Matararaba, S., Ishimura, T., Seeto, J., Rayawa, S., Kuruyawa, S., Nasila, A., Oloni, B., Ram, A.R., Saunivalu, P., Sing, P and Tegu, E. 2004b. Early Lapita settlement site at Bourewa, southwest Viti Levu Island, Fiji. Archaeology in Oceania 39: 139-143. 
Nunn, P.D., Pepe, C., Matararaba, S., Kumar, R., Sing, P., Nakoro, E., Gwilliam, M., Heorake, T., Kuilanisautabu, L., Nakoro, E., Narayan, L., Pastorizo, M.A., Robinson, S., Saunivalu, P. and Tamani, F. 2005. Human occupations of caves of the Rove Peninsula, southwest Viti Levu Island, Fiji. South Pacific Journal of Natural Science 23: 16-23.

Nunn, P.D., Matararaba, S., Kumar, R., Pene, C., Yuen, L., Pastorizo, M.A. 2006. Lapita on an island in the mangroves? The earliest human occupation at Qoqo Island, Southwest Viti Levu, Fiji. Archaeology in New Zealand 49: 205-212.

O'Day, S. Jones, O'Day, P., and Steadman, D.W. 2003. Defining the Lau context: Recent findings on Nayau, Lau Islands, Fiji. New Zealand Journal of Archaeology 25: 31-56.

Palmer, B. 1965. Excavations at Karobo, Viti Levu. New Zealand Archaeological Association Newsletter 8: 26-33.

Palmer, B. 1966. Lapita style potsherds from Fiji. Journal of the Polynesian Society 75: 373-377.

Palmer, B. 1967. Sigatoka research project. New Zealand Archaeological Association Newsletter 10: 2-15.

Palmer, B. 1969. Ring-ditch fortifications on windward Viti Levu, Fiji. Archaeology and Physical Anthropology in Oceania 4: 181-197.

Palmer, B. 1971. Naga ceremonial sites in the Navosa Upper Sigatoka valley. Final Report No. 1. Records of the Fiji Museum 5: 92-106.

Parry, J. 1987. The Sigatoka valley - pathways into prehistory. Bulletin of the Fiji Museum No. 9.

Petchey, F. 1995. Archaeology of Kudon: Archaeological analysis of Lapita ceramics from Mulifanua, Samoa and Sigatoka, Fiji. Unpublished MA thesis, Department of Anthropology, University of Auckland.

Prescott, J.R., Robertson, G.B. and Green, R.C. 1982. Thermoluminescence dating of Pacific island pottery: Successes and failures. Archaeology in Oceania 17: 142-147.

Rafter, T.A., Jansen, H.S., Lockerbie, L. and Trotter, M.M. 1972. New Zealand radiocarbon reference standards. In: Rafter, T.A. and Grant-Taylor, T. (eds), Proceedings of the Eighth International Radiocarbon Dating Conference, pp. 625-668. Royal Society of New Zealand, Wellington.

Rechtman, R.B. 1992. The evolution of socio-political complexity in the Fiji Islands. PhD thesis, Ann Arbor, Michigan.

Rosenthal, M.E. 1995. The archaeological excavation of an outrigger canoe from the Nasilai site, Rewa Delta, Viti Levu, Fiji. Asian Perspectives 34: 91-118.

Sand, C. 1997. The chronology of Lapita ware in New Caledonia. Antiquity 71: 539-547.

Sand, C. and Valentin, F. 1997. Cikobia et Naqelelevu. Programme archeologique et anthropologique sur deux iles de Fidje. Resultats preliminaires de la mission 1997. Noumea, New Caledonia.

Specht, J. and Gosden, C. 1997. Dating Lapita pottery in the Bismarck Archipelago, Papua New Guinea. Asian Perspectives 36(2): 175-199.

Spriggs, M. 1990. Dating Lapita: another view. In Spriggs, M. (ed), Lapita design, form and composition, pp. 83-122. Occasional Papers in Prehistory, No. 20. Canberra, Department of Prehistory, Australian National University.

Stuiver, M. and Braziunas, T.F. 1993. Modeling atmospheric 14C influences and 14C ages of marinesamples to 10,000 BC. Radiocarbon 35: 137-189.

Stuiver, M. and Reimer, P.J. 1993. Extended 14C data base and revised CALIB 3.0 14C age calibration program. Radiocarbon 35: 215-230.

Thomas, F.R., Nunn, P.D., Osborne, T., Kumar, R., Areki, F., Matararaba, S., Steadman, D. and Hope G. 2004. Recent archaeological findings at Qaranilaca Cave, Vanuabalavu Island, Fiji. Archaeology in Oceania 39: 42-49.

Toggweiler, J.R., Dixon, K. and Broecker, W.S. 1991. The Peru upwelling and the ventilation of the South Pacific thermocline. Journal of Geophysical Research 96: 467-497.

White, P.J., Clark, G. and Bedford, S. 2000. Distribution, present and past, of Rattus praetor in the Pacific and its implications. Pacific Science 54: 105-117. 